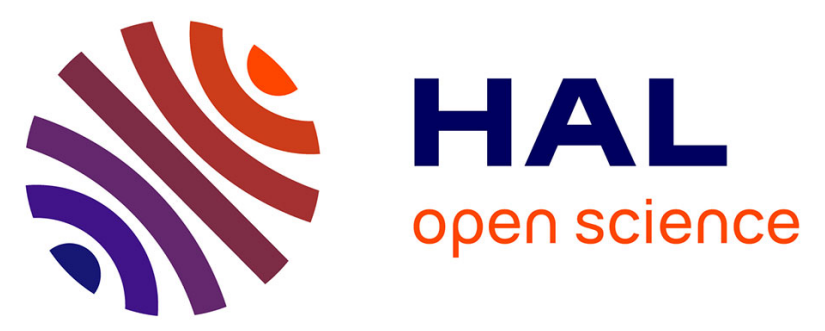

\title{
Biosynthesis of a broad-spectrum nicotianamine-like metallophore in Staphylococcus aureus
}

Ghassan Ghssein, Catherine Brutesco, Laurent Ouerdane, Clémentine Fojcik, Amélie Izaute, Shuanglong Wang, Christine Hajjar, Ryszard Lobinski, David Lemaire, Pierre Richaud, et al.

\section{To cite this version:}

Ghassan Ghssein, Catherine Brutesco, Laurent Ouerdane, Clémentine Fojcik, Amélie Izaute, et al.. Biosynthesis of a broad-spectrum nicotianamine-like metallophore in Staphylococcus aureus. Science, 2016, 352 (6289), pp.1105-1109. 10.1126/science.aaf1018 . hal-03133612

\section{HAL Id: hal-03133612 https://hal.science/hal-03133612}

Submitted on 13 Feb 2021

HAL is a multi-disciplinary open access archive for the deposit and dissemination of scientific research documents, whether they are published or not. The documents may come from teaching and research institutions in France or abroad, or from public or private research centers.
L'archive ouverte pluridisciplinaire HAL, est destinée au dépôt et à la diffusion de documents scientifiques de niveau recherche, publiés ou non, émanant des établissements d'enseignement et de recherche français ou étrangers, des laboratoires publics ou privés. 
Title: Biosynthesis of a broad-spectrum nicotianamine-like metallophore in Staphylococcus aureus

Authors: Ghassan Ghssein ${ }^{1,2,3, *}$, Catherine Brutesco ${ }^{1,2,3, *}$, Laurent Ouerdane ${ }^{4, *}$, Clémentine Fojcik $^{5}$, Amélie Izaute ${ }^{1,2,3}$, Shuanglong Wang ${ }^{4}$, Christine Hajjar ${ }^{1,2,3}$, Ryszard Lobinski ${ }^{4}$, David Lemaire $^{2,3,6}$, Pierre Richaud ${ }^{2,3,7}$, Romé Voulhoux ${ }^{8}$, Akbar Espaillat ${ }^{9}$, Felipe Cava ${ }^{9}$, David Pignol $^{1,2,3}$, Elise Borezée-Durant ${ }^{5}$, Pascal Arnoux ${ }^{1,2,3, \dagger}$

\section{Affiliations:}

$9{ }^{1}$ CEA, DSV, IBEB, Laboratoire de Bioénergétique Cellulaire, Saint-Paul-lez-Durance, France

$10 \quad{ }^{2}$ CNRS, UMR 7265 Biologie Végétale et Microbiologie Environnementales, Saint-Paul-lez-

11 Durance, France-

$12{ }^{3}$ Aix Marseille Université, CEA, CNRS, BVME UMR 7265, 13108, Saint Paul-Lez-Durance,

\section{France}

$14{ }^{4}$ CNRS-UPPA, Laboratoire de Chimie Analytique Bio-inorganique et Environnement,

15 UMR5254, Hélioparc, 2, Av. Angot, 64053 Pau, France

$16{ }^{5}$ Micalis Institute, INRA, AgroParisTech, Université Paris-Saclay, 78350 Jouy-en-Josas, France

$17{ }^{6}$ CEA, DSV, IBEB, Lab Interact Protein Metal, Saint-Paul-lez-Durance, France

$18{ }^{7}$ CEA, DSV, IBEB, Lab Bioenerget Biotechnol Bacteries \& Microalgues, Saint-Paul-lez-

19 Durance, France

$20 \quad{ }^{8}$ CNRS \& Aix-Marseille Université, Laboratoire d'Ingénierie des Systèmes Macromoléculaires

21 (UMR7255), Institut de Microbiologie de la Méditerranée (IMM), Marseille, France

$22{ }^{9}$ Laboratory for Molecular Infection Medicine Sweden, Department of Molecular Biology, Umeå

23 Centre for Microbial Research, Umeå University, Umeå, Sweden

25 *These authors contributed equally to this work.

$26 †$ Correspondence to: pascal.arnoux@cea.fr. 
1 Abstract: Metal acquisition is a vital microbial process in metal-scarce environments such as 2 inside a host. Using metabolomic exploration, targeted mutagenesis, and biochemical analysis, 3 we discovered an operon in Staphylococcus aureus that encodes the different functions required 4 for the biosynthesis and trafficking of a broad-spectrum metallophore related to plant 5 nicotianamine (here called staphylopine). The biosynthesis of staphylopine reveals the 6 association of three enzyme activities: a histidine racemase, an enzyme distantly related to 7 nicotianamine synthase, and a staphylopine dehydrogenase belonging to the DUF2338 family. 8 Staphylopine is involved in nickel, cobalt, zinc, copper and iron acquisition, depending on the 9 growth conditions. This biosynthetic pathway is conserved across other pathogens, thus 10 underscoring the importance of this metal acquisition strategy in infection. 


\section{Main Text:}

Metals are required in many life processes and, consequently, all organisms have developed mechanisms for their uptake and homeostasis. Pathogenic bacteria must face an additional barrier of metal limitation that is usually set by the host, precisely in order to prevent bacterial growth (1). This strategy of "nutritional immunity" by the hosts also extends to many micronutrients such as iron, manganese or zinc with dedicated mechanisms of sequestration (2, $3)$. The paradigm of metal acquisition in metal-deprived environments is associated with iron: whereas one strategy consists in transporting the reduced form, the most common scheme involves the synthesis of high affinity ferric siderophores, their extracellular release prior to capture and import of the siderophore- $\mathrm{Fe}^{3+}$ complex (4). These siderophores are frequently associated with virulence and may also be used for the transport of metals other than iron $(5,6)$, with only a few examples of other types of microbial metallophores. Methanobactin, for example, is a peptide-based chalkophore used for copper scavenging by methane oxidizing bacteria (7). Free L-Histidine is used as a nickelophore by some bacteria, and more complex molecules are possibly used by $S$. aureus or $H$. pylori, although their identity or functional relevance are unknown $(8,9)$. Recently, a nickel/cobalt uptake system (called CntA-F) was uncovered in $S$. aureus and was found to play a role in the virulence of this strain (10).

Plants have also evolved their own metal chelators and among them, nicotianamine is an important metabolite that is essential in the homeostasis of iron, copper, nickel and zinc $(11,12)$. Nicotianamine is also the first precursor of phytosiderophores, a family of molecules chemically different but functionally equivalent to siderophores (13). Nicotianamine is enzymatically synthesized by nicotianamine synthase (NAS), which catalyzes the condensation of three $\alpha$ aminobutyric acid moieties from S-adenosylmethionine (SAM) with the cyclization of one of them to form an azetidine ring (14-16). Nicotianamine and its phytosiderophore derivatives seem to be restricted to plants and some fungi, although some archaea apparently produce a nicotianamine-related molecule called thermonicotianamine, of which the functional role remains unknown (17).

Trying to better understand this family of plant NAS and archaeal NAS-like enzymes, we found a gene putatively coding for a NAS-like enzyme in bacterial genome, including $S$. aureus. The NAS-Like gene from $S$. aureus (sav2469), here called cntL, shares $12 \%$ sequence identity with one of the four NAS from Arabidopsis thaliana, which corresponds to a very distant homology. However, threading-based structure prediction programs detected a structural homology of CntL with eukaryotic NAS and archaeal NAS-like enzymes (Fig. S1). The gene coding for CntL is systematically located upstream of a gene whose product belongs to the DUF2338 family (cntM in S. aureus, sav2468; Figs. 1, S2). Threading-based prediction indicates with high confidence that this family of protein is related to Octopine-dehydrogenase, a $\mathrm{NAD}(\mathrm{P}) \mathrm{H}$ dependent enzyme which reductively condensates a free amino acid, such as arginine, with an $\alpha$-ketoacid, such as pyruvate (18). This family of enzymes is known to be involved in the escape of scallops from their starfish predators (19), or in the growth of parasitic bacteria, such as Agrobacterium tumefaciens at the expense of plants in which they induce opine-producing tumors, offering bacteria a selective growth advantage (20). In S. aureus, the gene upstream of cntL (cntK, sav2470) appears restricted to the phylum of Firmicutes and encodes a protein that is distantly related to a diaminopymelate epimerase (DapF). 
On the basis of these predictions, the putative activity of each of these three enzymes could be joined together to form a biosynthetic machinery: the $\mathrm{CntK}$ epimerase would produce an amine substrate of proper stereochemistry that would be modified by the CntL NAS-like enzyme through addition of one or two $\alpha$-aminobutyric acid moiety(ies), and CntM would reductively condensate an $\alpha$-ketoacid to the previously formed product, leading to the formation of a candidate molecule. Although their putative activity was not recognized at the time, the three corresponding genes (cntKLM) were shown to belong to an operonic structure involved in the import of nickel and cobalt ions through an $\mathrm{ABC}$ transporter named $c n t A-F$ ( 8 , Fig. 1A). Two independent genetic studies targeting either the solute binding protein (SBP; cntA) or the MFS transporter (Major Facilitator Superfamily, here called $c n t E$ ) indicated that this operon is important for the virulence and fitness of $S$. aureus $(10,21)$. The phenotype of these mutations could be reconciled by the hypothesis of a siderophore-like system with a biosynthetic machinery (CntKLM), a putative exporter (CntE) and a putative importer of a metalmetallophore complex (CntABCDF).

We attempted to identify the candidate metallophore through an ab-initio approach using $E$. coli or $S$. aureus strains, and through a knowledge-based approach using purified proteins (22). For the first approach we cloned the entire $10 \mathrm{~kb}$ cnt operon (cntKLMABCDFE) in E. coli and induced gene expression and protein production. After adding nickel we tracked small molecules co-eluting with nickel by metabolomic studies using hydrophilic interaction liquid chromatography (HILIC; Fig. S3). We also constructed a mutant strain of S. aureus devoid of the cntL gene and cultivated both the WT and this mutant strains in chemically defined media (CDM), a media with low metal concentration in which expression of the operon is known (10). We then detected metal complexes in the extracellular fractions by coupling HILIC separation with inductively coupled plasma-mass spectrometry (ICP-MS) and electrospray ionization-mass spectrometry (ESI-MS) (Fig. 1). A main peak was found to elute between 30 to $33 \mathrm{~min}$ in the HILIC/ICP-MS experiment, with the retention time depending on the detected metal (Fig. 1B). In all the cases these peaks were absent in the $c n t L$ mutant strain, therefore corroborating our central hypothesis of the existence of a direct link between these metal complexes and the central NAS-like enzyme. Investigation of these metal complexes by coupling HILIC and ESI-MS led us to find a candidate molecule showing the characteristic metal isotopic patterns (Fig. 1C, Fig. S3). The retention times of these compounds in HILIC/ESI-MS match those of the metals elution found by HILIC/ICP-MS, therefore indicating that the same metal complexes were observed by the two techniques. The knowledge-based approach consisted in the "fishing" of the nickelmetallophore complex: we expressed and purified the SBP (CntA) from E. coli and incubated it in a supernatant of a cntA mutant of $S$. aureus grown in CDM media. We then supplemented this mix with nickel to force nickel-metallophore complex formation and binding to CntA. After repurification of the SBP and further analysis by MS we looked for the characteristic nickel isotopic pattern and found a candidate molecule with the same mass as found by the metabolomic approaches (Fig. S4). Using our set of accurate masses with molecular formula finder softwares we proposed an empiric formula of $\mathrm{C}_{13} \mathrm{H}_{19} \mathrm{~N}_{4} \mathrm{O}_{6}$-Metal.

We attempted to fit this formula by using simple rules derived from our proposed enzymes activities and putative substrates (22), and we finally propose the structure of a candidate molecule that would be the result of three biosynthetic enzyme activities: 1) racemization of L-histidine in D-histidine by $\mathrm{CntK}, 2$ ) nucleophylic attack of one $\alpha$ aminobutyric acid moiety from SAM onto D-histidine to produce an intermediate (noted xNA) 
by $\mathrm{CntL}$ and, 3) reductive condensation of the xNA intermediate with a molecule of pyruvate by CntM (Fig. 2A). Gas-phase fragmentation of zinc and nickel metal complexes confirmed the proposed structure of the discovered molecule (Fig. S5), which we name staphylopine to recall its origin in S. aureus and its inclusion in the opine family (23).

We then tested and refined this hypothetical biosynthetic pathway by cloning, expressing and purifying the three enzymes and by testing their respective activities (Figs. 2B-D, S6). First, we incubated CntK with L- or D-histidine and found that it indeed produces D-histidine from the $\mathrm{L}$ - stereoisomer and vice versa, therefore establishing $\mathrm{CntK}$ as a histidine racemase (Fig. 2B). Because co-incubation with L-histidine and competing concentration of either L-alanine or Lmethionine did not inhibit the histidine racemase activity, CntK appears specific for histidine (Fig. S7). CntK is therefore not a diaminopimelate epimerase as its sequence similarity could suggest, but rather represents a novel enzyme family of histidine racemase. With regard to CntL we determined a dissociation constant of $68 \mu \mathrm{M}$ for SAM, which corroborates our central hypothesis that CntL binds to SAM (Fig. S8). We then tested our prediction suggesting the binding of $\mathrm{NAD}(\mathrm{P}) \mathrm{H}$ to CntM by incubating either NADH or NADPH with the purified enzyme. This indicated a preference of the enzyme for NADPH, and fluorescence Resonance Energy Transfer (FRET) between the tryptophan residue of the protein (excitation $280 \mathrm{~nm}$, emission 340 $\mathrm{nm})$ and $\mathrm{NAD}(\mathrm{P}) \mathrm{H}(340 \mathrm{~nm}$ and $460 \mathrm{~nm}$, respectively) further revealed that CntM is specific for NADPH with a dissociation constant of about $50 \mu \mathrm{M}$ (Fig. 2C), a value that was confirmed using microcalorimetric titrations (Fig. S8). Therefore, CntM has the biochemical characteristics of members of the opine synthase enzyme family, as suggested by the results of threading programs.

We then investigated the combined enzymatic activities of $\mathrm{CntK}$, CntL and CntM through the use of a carboxyl- $\left[{ }^{14} \mathrm{C}\right]$ labeled SAM substrate. The use of this radiolabelled substrate allowed us to follow the $\alpha$-aminobutyric acid moiety of SAM after product separation by thin layer chromatography (TLC) and autoradiography. We found that $\left[{ }^{14} \mathrm{C}\right]$-labeled SAM alone gave one strong band with three bands of lower intensity, likely corresponding to SAM and some degradation products, such as, e.g. S-adenosyl-L-homocysteine (Fig. 2D). Interestingly, although the incubation of $\left[{ }^{14} \mathrm{C}\right]-\mathrm{SAM}$ with $\mathrm{CntL}$ and L-His did not change this SAM pattern, the incubation with $\mathrm{D}$-His led to a novel prominent band which migrated below the lowest SAM band (Fig. 2D). According to our model, this band corresponds to the intermediate called xNA. We then tested the effect of co-incubating both CntL and CntM enzymes together with their putative substrates: L- and D-His, pyruvate and NADPH (Fig. 2D, lanes 4 and 5). Here again the SAM pattern stayed unchanged using L-His. However, using D-His we could detect two novel bands: one corresponding to the previous intermediate $\mathrm{xNA}$, and one that migrated between the two upper bands of SAM and would correspond to staphylopine. This identification is further supported by the fact that the staphylopine band appears at the expense of the xNA intermediate, as expected in such a case. We also confirmed the histidine racemase activity of CntK by coincubating all three enzymes together with their substrates. In this case, the incubation with L- or D-His both led to the appearance of the xNA and staphylopine bands, in accordance with the CntK activity, which produces both L- and D-His when supplied with either stereoisomer (Fig. 2D, lanes 6 and 7). Incubation of the three enzymes with different aminoacids, $\alpha$-keto acids and reducing agents confirms that the histidine racemase $\mathrm{CntK}$ is indeed specific for histidine and that CntM could use neither NADH nor $\alpha$-keto glutarate in place of NADPH and pyruvate, respectively (Fig. S9). Overall, these in vitro studies of the three biosynthetic enzymes show that 
they sequentially synthesize staphylopine according to our proposed scheme using common and defined substrates.

Finally, we confirmed that staphylopine biosynthesis is impaired in the cntL mutant of $S$. aureus and explored the effect of mutations of several genes in the cnt operon on metal homeostasis and staphylopine production and trafficking (Fig. 3). When compared to WT strain of $S$. aureus, both the xNA and the staphylopine bands disappeared in the cntL mutant, confirming that both bands are dependent on CntL activity (Fig. 3A). Furthermore the bands corresponding to xNA and staphylopine migrated at the same distance in E. coli or $S$. aureus cell extracts, therefore confirming that both molecules are produced heterologously in E. coli. We next sought to determine the effect of the cntL mutation with regard to intracellular metal accumulation in various culture conditions: CDM, where metal concentration is rather low, Chelex-demetalated CDM (dCDM) where metal level is even lower, NTA-demetalated CDM supplemented with metalated NTA resin to supposedly mimic a chelating environment ( $\mathrm{rCDM}$ ), and LB where metal concentration is high. We note that the growth of the cntL mutant is nearly identical to that of the WT strain in these media (Fig. S10), therefore indicating that metal content would be attributable to the absence of staphylopine and not to a growth defect. In all these culture conditions we established that the $c n t$ genes, transcribed from two promoters upstream $c n t K L M$ and $c n t A-E$, are highly expressed in metal scarce media, whereas their activities is lower but still significant in LB (Fig. S11). In CDM and dCDM, the cntL mutation caused a significant decrease in intracellular accumulation of nickel, copper and cobalt as determined by ICP-MS (Figs. 3B, S12). This metal accumulation defect is comparable to the one observed in the cntA-F mutant, indicating that metal or metal-staphylopine complexes are transported via CntA (Fig. 3B). This defect in metal accumulation is complemented when reintroducing the cnt $L$ gene in the mutant, although using slightly different conditions adapted for ICP-AES metal content determination (Fig. S13). In this case there is no significant decrease in intracellular copper, but a significant decrease in intracellular zinc, nickel and cobalt. In rCDM, the tendency observed in CDM is confirmed, although in this condition iron, zinc, copper, nickel and cobalt content are all affected in the mutant strain as compared to the WT. Finally, in a metal rich medium such as LB, the cntL deletion mutant accumulated less iron, copper and cobalt. Taken together, these sets of experiments suggest that, depending on the nature of the metal, its concentration, and the bacterial growth status, staphylopine is involved in the import of a large array of divalent metals in S. aureus. Testing metal transport over 10 minutes supports the involvement of both staphylopine and CntA-F in the import of all these metals. Indeed, import of iron, zinc, nickel and cobalt are all significantly decreased in both cntL and cntA-F mutants (Fig. 3C). The significant short-term transport of iron and zinc is consistent with their known regulatory effect exerted on the cnt operon $(10,21)$. The transport of copper is somehow different as WT cells efficiently export this metal during this short time, probably in response to its toxicity. In this case the absence of CntL or CntA-F appears to decrease this toxicity and its consecutive efflux. In order to investigate the transport of metal-staphylopine complexes through CntA, we exploited the sensitivity of $S$. aureus to high cobalt concentration. We found that $c n t L$ and $c n t A-F$ mutant strains are resistant to an otherwise toxic concentration of cobalt to WT, whereas supplementation of synthetic staphylopine in the media restores its toxicity in cntL but not in cntA-F strain (Fig. S14). This suggests a co-transport of cobaltstaphylopine complexes via CntA-F, as the $\mathrm{ABC}$ transporter is still present in the cntL (hence the cobalt toxicity) whereas it is absent in the cntA-F mutant (where cobalt-staphylopine cannot enter the cell). Finally, we measured the staphylopine level in the intracellular and supernatant 
fractions of the WT, cntABCDF, cntL and $c n t E$ mutants (Fig. 3D). This experiment confirmed the absolute need of CntL in the biosynthesis of staphylopine, further supported the function of CntA-F in the import of staphylopine-metal complexes, and clearly indicated that CntE is involved in the export of staphylopine. Overall, despite being distantly related, staphylopine apparently mirrors the effect that nicotianamine has on the same range of metal but in the context of metal homeostasis and transport in plants (17). Chemically synthesized staphylopine has the same range of metal affinity than nicotianamine (similar $K_{\mathrm{d}}$ and affinity order: $\mathrm{Cu}^{2+}>\mathrm{Ni}^{2+}>$ $\mathrm{Co}^{2+}>\mathrm{Zn}^{2+}>\mathrm{Fe}^{2+}$; Fig. S15), further supporting their functional comparison as broad-spectrum metallophores.

The mechanisms of staphylopine biosynthesis export and recovery of the metal complexes are summarized in Fig. 4. The biosynthesis of staphylopine aggregates three unprecedented enzyme activities with the use of D-histidine, the fusion of a single $\alpha$ aminobutyric acid moiety from SAM, and the fabrication of an opine derivative. This latter activity was so far restricted to plant's bacterial transfection or anaerobic glycolysis in marine invertebrates $(19,23)$. The use of histidine as a building block is interesting as the free amino acid has a relatively high affinity for nickel on its own and is directly used for nickel uptake by bacteria $(8,24-26)$. However, the use of the D- stereoisomer of histidine is surprising because Damino acids are mainly used in cell wall biosynthesis and have been mostly restricted to alanine and glutamate (27), although other "non-canonical" D-amino acids are reported to be involved in the composition of the cell wall $(28,29)$, in biofilm stability $(30)$, virulence $(31)$ or sporulation (32). Another peculiarity of the staphylopine biosynthetic machinery resides in the NAS-like CntL enzyme that only uses one $\alpha$-aminobutyric acid moiety from SAM, whereas the NAS-like from $M$. thermotautrophicum uses two, and plant enzymes use three such moieties. Interestingly, the evolution from bacterial staphylopine to plant's nicotianamine preserves a large part of the molecule's backbone, therefore suggesting a conservative chemical evolution going together with enzymatic simplifications wherein plant nicotianamine is synthesized by a single enzyme. Finally, homologous genes involved in staphylopine biosynthesis are conserved in other pathogenic bacteria such as Yersinia pestis or Pseudomonas aeruginosa underscoring the likely influence of this metallophore in bacterial pathogenicity. Indeed, the export pathway of this metallophore was recently identified as a critical function for the growth of $P$. aeruginosa in airway mucus secretions where metal access is severely limited (33). 


\section{References and Notes:}

2 1. M. L. Reniere, G. Pishchany, E. P. Skaar, in Iron uptake and homeostasis in

3 microorganisms. (2010).

4 2. B. D. Corbin et al., Science 319, 962 (2008).

5 3. M. I. Hood, E. P. Skaar, Nat Rev Microbiol 10, 525 (2012).

6 4. Z. Ma, F. E. Jacobsen, D. P. Giedroc, Chem Rev 109, 4644 (2009).

7 5. A. G. Bobrov et al., Mol Microbiol 93, 759 (2014).

8 6. I. J. Schalk, M. Hannauer, A. Braud, Environ Microbiol 13, 2844 (2011).

9 7. H. J. Kim et al., Science 305, 1612 (2004).

10 8. H. Lebrette et al., Metallomics 7, 613 (2015).

$119 . \quad$ K. Schauer, B. Gouget, M. Carriere, A. Labigne, H. de Reuse, Mol Microbiol 63, 1054 12 (2007).

13 10. L. Remy et al., Mol Microbiol 87, 730 (2013).

14 11. C. Curie et al., Ann Bot 103, 1 (2009).

15 12. E. L. Walker, B. M. Waters, Curr Opin Plant Biol 14, 318 (2011).

16 13. R. Hell, U. W. Stephan, Planta 216, 541 (2003).

17 14. A. Herbik et al., Eur J Biochem 265, 231 (1999).

18 15. K. Higuchi et al., Plant Physiol 119, 471 (1999).

16. H. Q. Ling, G. Koch, H. Baumlein, M. W. Ganal, Proc Natl Acad Sci U S A 96, 7098 (1999).

17. C. Dreyfus, D. Lemaire, S. Mari, D. Pignol, P. Arnoux, Proc Natl Acad Sci U S A 106, 16180 (2009).

18. S. H. Smits, A. Mueller, L. Schmitt, M. K. Grieshaber, J Mol Biol 381, 200 (2008).

19. M. Harcet, D. Perina, B. Plese, Biochem Genet 51, 666 (2013).

20. M. W. Bevan, M. D. Chilton, Annu Rev Genet 16, 357 (1982).

21. Y. Ding, Y. Fu, J. C. Lee, D. C. Hooper, J Bacteriol 194, 6586 (2012).

22. Materials and methods available on Science online.

23. J. Thompson, J. A. Donkersloot, Annu Rev Biochem 61, 517 (1992).

24. P. T. Chivers, E. L. Benanti, V. Heil-Chapdelaine, J. S. Iwig, J. L. Rowe, Metallomics 4, 1043 (2012).

25. H. Lebrette, M. Iannello, J. C. Fontecilla-Camps, C. Cavazza, J Inorg Biochem 121, 16 (2013).

26. M. M. Shaik, L. Cendron, M. Salamina, M. Ruzzene, G. Zanotti, Mol Microbiol 91, 724 (2014).

27. W. Vollmer, D. Blanot, M. A. de Pedro, FEMS Microbiol Rev 32, 149 (2008).

28. F. Cava, M. A. de Pedro, H. Lam, B. M. Davis, M. K. Waldor, Embo J 30, 3442 (2011).

29. H. Lam et al., Science 325, 1552 (2009).

30. I. Kolodkin-Gal et al., Science 328, 627 (2010).

31. A. T. Anfora, B. J. Haugen, P. Roesch, P. Redford, R. A. Welch, Infect Immun 75, 5298 (2007).

32. K. A. O'Connor, D. R. Zusman, Mol Microbiol 24, 839 (1997).

33. M. Gi et al., Sci Rep 5, 14644 (2015). 
1 Acknowledgments: We thank the Commissariat à l'Energie Atomique et aux Energies 2 Alternatives (CEA), the Agence Nationale de la Recherche (ANR) and the CEA program 3 ToxNuc-E for financial support. Support was also provided by the HélioBiotec platform (funded 4 by the European Regional Development Fund, the Région Provence Alpes Côte d'Azur, the 5 French Ministry of Research, and the "Commissariat à l'Energie Atomique et aux Energies 6 Alternatives"). G. G. was supported by the Région PACA. Work at the F. C. lab was supported 7 by MIMS, KAW, the Swedish Research Council and a Kempe foundation scholarship to A. E. 8 We wish to thank J. Lavergne for careful reading of the manuscript and we also thank A. 9 Verméglio, C. Cavazza and H. Lebrette for fruitful discussions. 
Figure and Figure Legends

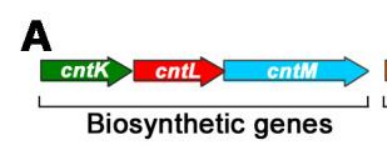

B
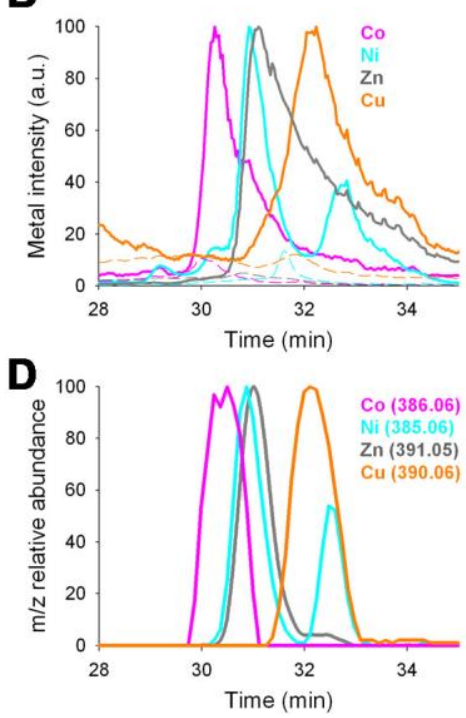



C

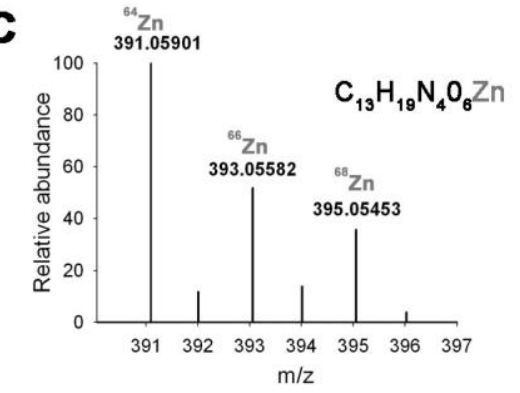

$\mathbf{E}$
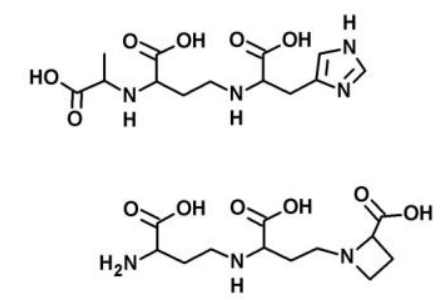

Fig. 1. The cnt operon and detection of a broad-spectrum metallophore. (A) The cnt operon encodes three putative biosynthetic genes together with the genes coding for a full ABC transporter and a transporter belonging to the MFS family. (B) HILIC/ICP-MS chromatograms of extracellular fractions of $S$. aureus and comparison of the WT strain (plain line) with the cntL mutant (dotted lines). (C) Mass spectra of a chelating compound present in its free and complexed forms (here shown in complex with $\mathrm{Zn}$ ) in the extracellular fraction of the WT strain but absent in the cntL mutant as observed in chromatogram around $31 \mathrm{~min}$ during HILIC/ESIMS analyses. The empirical molecular formula was deduced from exact masses. (D) HILIC/ESIMS extracted ion chromatograms (XIC) of some metal-ions observed to form a complex with the above-mentioned chelator. Chromatograms were traced using the exact masses $( \pm 3 \mathrm{ppm})$ of the 13 expected complex and these masses were absent in the extracellular fraction of the cntL mutant. 14 (E) Proposed chemical structure of staphylopine (top) and comparison with nicotianamine 15 (bottom). 
A
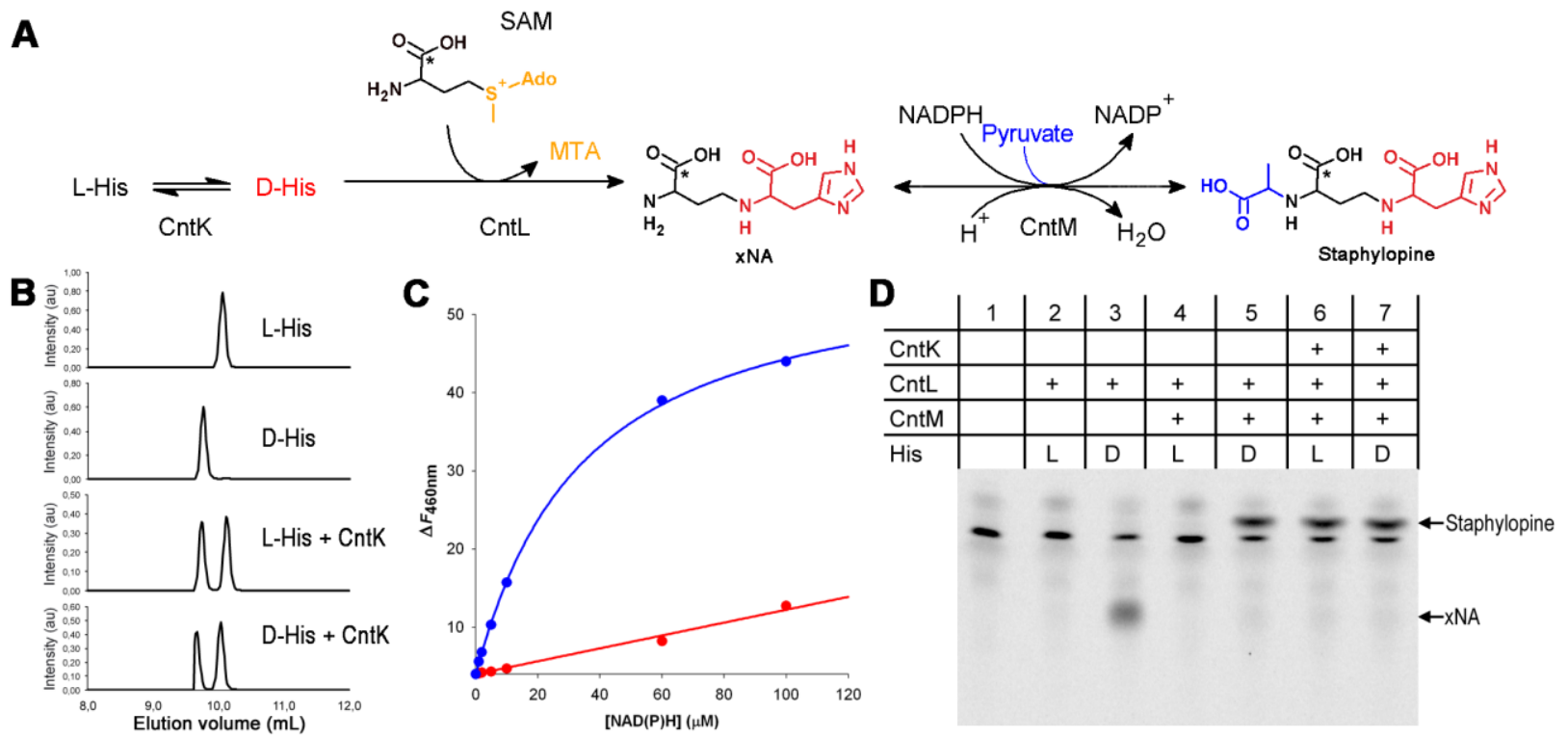

2 Fig. 2. Biosynthesis of staphylopine and biochemical characterization of the biosynthetic 3 enzymes. (A) Biosynthesis pathway for the assembly of staphylopine from L-histidine, SAM and 4 pyruvate. (B) Epimerase assay of CntK and comparison with L- and D-histidine standards. (C) 5 Titration of NADH (red) and NADPH (blue) binding to CntM ( $5 \mu \mathrm{M})$ followed by fluorescence 6 energy transfer between tryptophan excitation $(280 \mathrm{~nm})$ and $\mathrm{NAD}(\mathrm{P}) \mathrm{H}$ emission $(460 \mathrm{~nm})$. Fitting of the data obtained for NADPH binding lead to a $\mathrm{K}_{\mathrm{d}}$ of $50 \mu \mathrm{M}$. (D) TLC separation of reaction products incubating $\left[{ }^{14} \mathrm{C}\right]-\mathrm{SAM}$ (labeled on its $\alpha$-aminobutyric acid moiety; marked with a $*$ in panel A) with various purified enzymes, pyruvate, NADPH and either L- or D10 Histidine. 




2 Fig. 3. Effect of cnt mutations in metal content and staphylopine localization. (A) TLC 3 separation of co-incubation of E. coli cell extracts separately expressing CntK, CntL and CntM 4 with $\left[{ }^{14} \mathrm{C}\right]-\mathrm{SAM}$ and comparison with the cell extracts from WT $S$. aureus and cntL mutant 5 strains. (B) Intracellular metal content of the $S$. aureus WT (left), cntL (middle) and cntA-F 6 (right) strains grown in CDM, and determined by ICP-MS. Error bars, mean \pm s.d. $* * P<0.01$ and $7 \quad * * * P<0.001$. (C) Intracellular metal content change of the $S$. aureus WT (left), cntL (middle) and 8 cntA-F (right) strains grown in dCDM until late exponential phase (T0) and then incubated 10 9 minutes with $0.3 \mu \mathrm{M} \mathrm{Fe}(\mathrm{II}), \mathrm{Zn}$, and $0.1 \mu \mathrm{M} \mathrm{Mn}, \mathrm{Ni}, \mathrm{Co}, \mathrm{Cu}$. Metal content variation was 10 determined by ICP-MS and expressed as the differential of atoms after $10 \mathrm{~min}$. as compared to 11 T0. Error bars, mean \pm s.d. $* P<0.05$, $* * P<0.01$ and $* * * P<0.001$. (D) Staphylopine level in the 12 intracellular (left; note the logarithm scale) and supernatant (right) fractions of the WT, $13 c n t A B C D F$, cntL and $c n t E$ mutant strains. Error bars, mean \pm s.d. $* P<0.05$ and $* * P<0.01$, ND: 14 Not detected. 

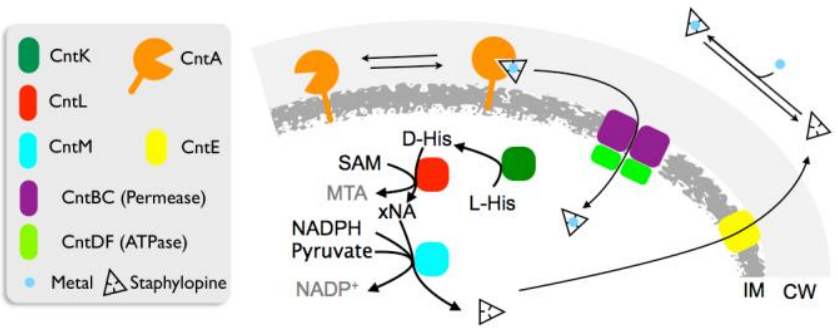

Figure 4: Model of staphylopine biosynthesis, export and recovery of staphylopine-metal complexes through the ABC transporter. IM: Inner Membrane, CW: Cell Wall. 


\section{Supplementary Materials}

2 Materials and Methods

3 Figs. S1-S16

4 Tables S1-S3

5 Refs (34-49)

6 
Supplementary Materials:

2 Materials and Methods

3 Bioinformatic analysis

4 Sequences from CntK (encoded by sav2470), CntL (sav2469) and CntM (sav2468) were analyzed by searching for homologues using PsiBLAST (34) and the Pfam database (35). Sequence alignment was done using the Muscle program (36) and visualized in the Jalview suite (37). The threading program Phyre2 (38) was used to detect distant homologues. Gene syntheny were inspected using the MaGe MicroScope web interface (39).

Gene cloning, protein expression and purification

11 The entire operon from $S$. aureus (9,3 kb; from cntK to $c n t E$; sav2470 to sav2462) was amplified by PCR (See Table S1 for oligonucleotides used in this study). The resulting fragment was digested by NdeI and XhoI and ligated in plasmid pET22b+. The three biosynthetic genes (cntK, cntL and $c n t M$ ) and the cntA gene were separately cloned in pET100, pET-SUMO, pET101 and pET100, respectively, according to standard protocols. After co-transformation with plasmid pRARE (encoding for rare codon in E. coli BL21) strains were cultivated in LB media supplemented with appropriate antibiotics. When the OD of the culture was about 0.6 they were transferred at $16{ }^{\circ} \mathrm{C}$ and protein expression was induced by adding $0.1 \mathrm{mM}$ IPTG. Bacteria were recovered after overnight growth by centrifugation at 7,000 $\mathrm{g}$ for $15 \mathrm{~min}$. Cells were resuspended in buffer $\mathrm{A}\left(20 \mathrm{mM} \mathrm{Na}, \mathrm{K} \mathrm{PO}_{4}(\mathrm{pH} 7.5), 500 \mathrm{mM} \mathrm{NaCl}, 20 \mathrm{mM}\right.$ imidazole) and disrupted using a French press operating at $1000 \mathrm{psi}$. Cell debris were removed by centrifugation at $8,000 \mathrm{~g}$ for 20 min. The supernatant was centrifuged at $100,000 \mathrm{~g}$ for $1 \mathrm{~h}$ at $4^{\circ} \mathrm{C}$ to remove cell wall debris and membrane proteins. The resulting soluble fraction was loaded on a nickel affinity column (HisTrap $1 \mathrm{ml}$ column, G.E. Healthcare), and the protein was eluted stepwise with imidazole (75 $\mathrm{mM}$ wash and $250 \mathrm{mM}$ elution). Collected fractions were loaded onto a gel filtration column (Hiload 26/60 Superdex 200, G.E. Healthcare), equilibrated with buffer B (20 mM HEPES, 50 $\mathrm{mM} \mathrm{NaCl} \mathrm{pH}$ 8.0) for CntA, CntK and CntL, or buffer C (buffer B $+500 \mathrm{mM} \mathrm{NaCl}$ ) for CntM. Before gel filtration, the tag of CntL was removed by incubating the protein $(1 \mathrm{mg} / \mathrm{mL})$ with the SUMO (Ulp-1) protease using a protein / protease ratio of $1 / 100(\mathrm{w} / \mathrm{w})$ and an overnight incubation at room temperature. Protease, free tag and uncleaved protein were then removed by a second passage through a Ni-NTA column equilibrated with buffer A.

\section{Construction of cntL and cntE deletion mutants of $S$. aureus and complementation}

A deletion mutant of $S$. aureus RN6390 was obtained as described (40). Briefly, two DNA fragments corresponding to upstream and downstream regions of $c n t L$ or $c n t E$ gene were amplified from RN6390 chromosomal DNA using primers pairs listed in Table S1. Deletion fragments were generated using splicing by overlap extension PCR and cloned into BamHI (for cntL mutant) or BamHI and XmaI (for cntE mutant) sites of shuttle vector pMAD (41). The resulting plasmid was introduced into $S$. aureus RN4220 by electroporation, extracted and subsequently introduced into S. aureus RN6390. Deletion of the chromosomal region of interest was subsequently obtained by double-crossing over events as previously described (42). To complement the cntL deletion strain, the cntL gene was amplified by PCR (Table S1) and 
inserted into BamHI site of shuttle vector pOS1-Plgt to generate pCntL in which cntL is under the control of the lgt promoter (43). Recombinant vectors (pOS1-Plgt and pCntL) were introduced into $S$. aureus strains as described above.

Cell culture conditionsWT and mutant strains of $S$. aureus were grown at $37^{\circ} \mathrm{C}$ with aeration in either CDM (40, 44) deprived of added metals except magnesium (1 mM; Table S2), demetalated CDM (CDM treated with chelex; dCDM) or LB. For cultures in CDM with metalNTA resin (rCDM), NTA-treated CDM and metalated resin were prepared as follows: Ni-NTA His-bind resin (EMD Millipore, Billerica) was extensively washed with 100mM EDTA ( 15 time the resin volume) to eliminate nickel linked to NTA, and then with double distilled water to remove EDTA. CDM was then incubated overnight with the demetalated NTA resin and further filtrated to obtain NTA-treated CDM. The resin (now metalated by the traces of metals present in CDM) was then placed into Amicon centrifugal filter units (Amicon Ultra- $0.5 \mathrm{ml} 3 \mathrm{KDa}$; Merck Millipore, Tullagreen) inserted into eppendorf tubes. After having cut the bottom of these tubes, they were introduced into $50 \mathrm{ml}$ Falcon tubes containing the NTA-treated CDM. Despite demetalation by EDTA, the total amounts of metal in rCDM treated cells seems to indicate that residual amounts of $\mathrm{Mn}, \mathrm{Cu}$ and $\mathrm{Ni}$ remained trapped by the resin as the uptake of these metals was found higher in rMCD cells than CDM cells. For short time uptake experiments, strains were grown in demetalated CDM until late exponential phase, then supplemented with a metal cocktail $(0.3 \mu \mathrm{M} \mathrm{Fe}(\mathrm{II})$ and $\mathrm{Zn} ; 0.1 \mu \mathrm{M} \mathrm{Ni}, \mathrm{Co}, \mathrm{Cu}, \mathrm{Mn})$ and incubated for 10 minutes prior to metal level determination by ICP-MS.

Analysis of metal concentrations by ICP-MS

Metal content of the media used in this study are reported in Table S3. After growing until late exponential phase or overnight, the cultures $(15 \mathrm{~mL})$ were centrifuged and pellets were washed three times with ice cold $1 \mathrm{mM}$ EDTA and then once with double distilled water. Cells were then dried overnight at $100{ }^{\circ} \mathrm{C}$. Metal contents were determined in cell fraction by resuspending the dried pellet with a $200 \mu \mathrm{L}$ mixture of $70 \% \mathrm{HNO}_{3}(100 \mu \mathrm{L})$ and $30 \% \mathrm{H}_{2} \mathrm{O}_{2}(100 \mu \mathrm{L})$. The mixture was heated at $80^{\circ} \mathrm{C}$ for $3 \mathrm{~h}$ and then diluted with water to reach a final volume of $2 \mathrm{~mL}$. Acidified sample solutions were then analyzed by ICP-MS (Agilent 7500cs) operating in hydrogen collision gas mode $\left(\mathrm{H}_{2}\right)$ to remove possible interferences.

\section{Cell fractionation for staphylopine quantification and TLC assay}

WT and mutant strains of $S$. aureus were grown in $\mathrm{CDM}$ at $37^{\circ} \mathrm{C}$ with aeration, centrifuged, and the supernatants were collected, filtrated and stored at $-20^{\circ} \mathrm{C}$. The pellets were resuspended in 2 $\mathrm{ml}$ lysis buffer $(0.1 \mathrm{mg} / \mathrm{ml}$ lysostaphin in Hepes $20 \mathrm{mM})$ and incubated at $37^{\circ} \mathrm{C}$ for $1 \mathrm{~h}$ by shaking (150 rpm). Lysates were then transferred into tubes containing $0.1 \mathrm{~mm}$ glass beads (MP Biomedicals, Solon, $\mathrm{OH}$ ) and subjected to mechanical disruption by homogenization using a FastPrep FP120 instrument (Thermo Scientific, Waltham) in three $45 \mathrm{~s}$ intervals at a speed of 6.0 , with cooling on ice between intervals. The lysates were centrifuged at $14,000 \mathrm{~g}$ for $20 \mathrm{~min}$ at $4^{\circ} \mathrm{C}$ and supernatants were collected. Staphylopine level determination is described further below. 
This separation method preserves metal-ligand complexes and facilitates their downstream identification $(45,46)$. Analytical reagent grade chemicals such as acetonitrile, acetic acid, formic acid, nitric acid and ammonia were purchased from Sigma-Aldrich (Saint-QuentinFallavier, France). Ultrapure water (18 M $\Omega . c m)$ was obtained from a Milli-Q system (Millipore, Bedford, MA). Microbore HILIC separations were performed using an Agilent 1100 capillary HPLC system (Agilent, Tokyo, Japan) equipped with a $100 \mu 1 \mathrm{~min}^{-1}$ splitter module. ICP-MS detection was achieved using a model 7500cs instrument (Agilent) fitted with platinum cones, 1 $\mathrm{mm}$ i.d. injector torch and a T-connector allowing the introduction of $5 \% \mathrm{O}_{2}$. The HILIC/ICPMS coupling was done via an Isomist interface (Glass Expansion, Melbourne, Vic, Australia) consisting of a 20-ml Cinnabar cyclonic spray chamber cooled to $2{ }^{\circ} \mathrm{C}$ and fitted with a $50 \mu 1$ $\min ^{-1}$ Micromist U-series nebulizer.

The column used for HILIC separation was a TSK gel amide $80(250 \mathrm{~mm} \times 1 \mathrm{~mm}$ i.d., $5 \mu \mathrm{m})$ from Tosoh Biosciences (Stuttgart, Germany). Gradient elution, at a flow rate of $50 \mu 1 \mathrm{~min}^{-1}$, was carried out using eluent $\mathrm{A}$, acetonitrile, and eluent $\mathrm{B}, 5 \mathrm{mM}$ ammonium formate $(\mathrm{pH} 5.5)$. The gradient program was: $0-5$ min $10 \% \mathrm{~B}, 5-45$ min up to $50 \% \mathrm{~B}, 45-50 \mathrm{~min} 50 \% \mathrm{~B}, 50-52 \mathrm{~min}$ up to $65 \% \mathrm{~B}, 52-55 \min 65 \% \mathrm{~B}, 55-60$ min down to $10 \% \mathrm{~B}, 60-65 \mathrm{~min} 10 \% \mathrm{~B}$. Samples were diluted with acetonitrile and water to obtain a $1: 2$, sample to acetonitrile ratio, and centrifuged. A $7-\mu 1$ aliquot of the supernatant was injected into the HILIC column each time.

\section{Analysis for metal complexes using HILIC/ESI-MS}

For HILIC/ESI-MS, the HPLC systems were connected to an LTQ Orbitrap Velos mass spectrometer (Thermo Fisher Scientific, Bremen, Germany). The coupling was achieved via a heated electrospray ionization source (H-ESI II) (Thermo Fisher Scientific. The ion source was operated either in the positive ion mode at $3.0 \mathrm{kV}$. The vaporizer temperature of the source was set to $120{ }^{\circ} \mathrm{C}$ and the capillary temperature to $280{ }^{\circ} \mathrm{C}$. In full MS mode, the resolution was set at 100000 (FWHM at $\mathrm{m} / \mathrm{z}$ 400) whereas in MS/MS mode it was set at 30000 . MS data were processed using Xcalibur 2.1 software and MetWorks 1.2.1 software (Thermo Fischer Scientific) to screen spectra for metal containing molecules. To get accurate masses, MS and MS/MS spectra were recalibrated offline using precursor/fragment ions with known formula. Putative metal species were fragmented during a subsequent chromatographic run with collision induced dissociation (CID) mode at 35\% energy. In order to fit the molecular formula that we obtained from accurate masses, we used simple chemical rules as follow: $1 /$ each time there is a reductive condensation there is a mass loss of 16 Da between the two reactants. 2/ each time there is an $\alpha$ aminobutyric acid moiety transfer from SAM to an amino acid there is a mass loss of $2 \mathrm{Da}$ between the two reactants. This way, the "simplest" molecule would consist of pyruvate (88Da) reductively condensated with glycine $(75 \mathrm{Da})$ that would be modified by two $\alpha$-aminobutyric acid moieties (103 Da), which would give a molecular weight of $349 \mathrm{Da}$, above the $328 \mathrm{Da}$ measured. The only possibility is therefore to use only one $\alpha$-aminobutyric acid moiety from SAM; and only using histidine (155 Da) and pyruvate we can obtain the expected $328 \mathrm{Da}$. 
Synthetic staphylopine (N-[3-(N-L-Alanyl)-amino-3-carboxypropyl]-D-histidine) was ordered to Toronto Research Chemical Inc (Toronto, Canada) and its NMR spectrum is provided in Fig. S16. Metal content of the studied elements in staphylopine synthetic standard was measured by ICP MS and was found to be lower than $0.1 \%$ of staphylopine mass. Analysis of this synthetic standard by HILIC/ESI-MS showed that it has the same retention time, chromatographic peak shapes, fragmentation behavior in MS instrument and metal affinity than the natural molecule found in culture supernatants. It was then used also to quantify staphylopine in spent culture media through standard addition technique, which resulted in staphylopine peak increases during HILIC/ESI-MS analysis and which allow to determine staphylopine concentration and confirmed results obtained by HILIC/ICP-MS analysis. We tested metal affinity of staphylopine standard by mixing different metals ( $\mathrm{Fe}(\mathrm{II}), \mathrm{Fe}(\mathrm{III}), \mathrm{Cu}, \mathrm{Zn}, \mathrm{Co}, \mathrm{Ni}$ ) with this molecule prior to direct analysis by ESI-MS. It was found that metal affinity for staphylopine was in the following order: $\mathrm{Cu}$ (II) $>\mathrm{Ni}>\mathrm{Co}>\mathrm{Zn}>\mathrm{Fe}$ (II) $>\mathrm{Mn}$ (II), which is identical to what was observed for nicotianamine. Fe(III) affinity for staphylopine relative to other metals is $\mathrm{pH}$ dependent and, whereas it has a higher pKd value than $\mathrm{Cu}(\mathrm{II}), \mathrm{Fe}(\mathrm{III})$ has a poor affinity for staphylopine at neutral $\mathrm{pH}$, a phenomenon already described for nicotianamine (47). Stability constants for staphylopine:metal complexes were estimated by metal competition experiments with nicotianamine. Mixtures of nicotianamine:staphylopine:metal were analyzed in physiological conditions $(\mathrm{pH}=7)$ by infusion in ESI MS. Were analyzed: 1) a mixture of only nicotianamine and staphylopine at $100 \mu \mathrm{M}$ each to establish maximal signal intensity for these free ligands, 2) a mixture of nicotianamine $(100 \mu \mathrm{M})$, staphylopine $(100 \mu \mathrm{M})$ and an excess of metal $(1 \mathrm{mM}$, each metal was tested in a different experience) to establish maximal signal intensity for the complexed ligands and 3) a mixture of nicotianamine $(100 \mu \mathrm{M})$, staphylopine $(100 \mu \mathrm{M})$ and limited level of metal $(80 \mu \mathrm{M}$, each metal was tested in a different experience) to created competition and observed the proportion between metal complexes formed. The estimated $\mathrm{pKd}$ values are given in Fig. S15C. Like nicotianamine also, the complex of staphylopine with metal is only observed as a 1:1 complex during ESI MS analysis. All these data lead to consider that staphylopine is very similar to nicotianamine in terms of metal affinity and structure. A putative model for metal:staphylopine complex can then be proposed (hexadentate structure with 3 amino groups (the ones spaced by 3 carbons) and the 3 carboxylic groups, Fig. S15A).

\section{Fishing and Metabolomic studies}

The CntA protein, predicted to bind the metallophore-nickel complex, was purified by conventional HisTrap and incubated in $50 \mathrm{~mL}$ of a culture supernatant from a cntA mutant of $S$. aureus cultivated in CDM. Nickel was then added to a final concentration of $50 \mu \mathrm{M}$ to allow the nickel-metallophore complex to form and to bind to CntA. After $2 \mathrm{~h}$ of incubation the CntA protein was purified again, exchanged against a buffer containing $10 \mathrm{mM}$ ammonium acetate, and finally analyzed by MS. Cell fraction of an E. coli strain expressing the entire cnt operon was also analyzed by HILIC/ICP-MS for metal detection and HILIC/ESI-MS for metabolite and metal complex identification.

\section{Metal content using ICP-AES (atomic emission spectroscopy)}

43 S. aureus strains were grown at $37{ }^{\circ} \mathrm{C}$ with aeration in LB medium or in CDM until late 44 exponential phase. In CDM condition, nickel, copper, zinc or cobalt chloride was then added (1 
$\mu \mathrm{M}$ final). Cultures were then grown overnight and harvested by centrifugation at $6,000 \mathrm{~g}$ at $4^{\circ} \mathrm{C}$ for $15 \mathrm{~min}$. Pellets were washed three times with ice-cold $1 \mathrm{mM}$ EDTA and once in double distilled water. Cells were dried overnight at $100^{\circ} \mathrm{C}$ and then digested with $500 \mu \mathrm{l}$ of nitric acid (70 \% $\mathrm{HNO}_{3}$ ICP Grade, JT Baker) overnight at $80^{\circ} \mathrm{C}$. Samples were further diluted by addition of distilled water (final volume $6 \mathrm{~mL}$ ). The element measurements were performed with Inductively Coupled Plasma - Absorption Emission Spectrometer (ICP-AES Vista MPX, Varian). The metal concentrations were determined using standard curves obtained by analyzing ICP grade element standard solutions. The metal contents were calculated assuming $4 \times 10^{8}$ CFU (Colony Forming Unit) for an OD600 of 1 unit, as determined by plating diluted cultures grown in $\mathrm{CD}$ medium.

\section{Fluorescence studies and microcalorimetry}

Fluorescence studies were performed using a Cary Eclipse spectrophotometer (Agilent). The FRET experiment was done using a protein concentration of $5 \mu \mathrm{M}$ an excitation wavelength at $280 \mathrm{~nm}$. The signal was recorded between 400 and $500 \mathrm{~nm}$. Five spectra were averaged in order to increase signal to noise ratio. The dissociation constants were obtained by directly fitting the experimental data to a binding equation in SigmaPlot.

Microcalorimetry was used to determine the dissociation constants for the binding of NADPH to CntM using a MicroCal iTC200 system (MicroCal). The experiments were performed at $25^{\circ} \mathrm{C}$ in buffer C. Titration experiments consisted of 13 injections $(3 \mu \mathrm{L})$ of NADPH ( $1 \mathrm{mM}$ in buffer C) to $200 \mu \mathrm{L}$ of protein $(97 \mu \mathrm{M})$. Dissociation constant was obtained using one set of sites fitting in Origin (MicroCal).

\section{Epimerase/Racemase assay}

The product from a racemization reaction ( $30 \mu \mathrm{g}$ of purified $\mathrm{CntK}$ incubated during $30 \mathrm{~min}$ at room temperature in $100 \mathrm{~mL}$ of buffer $(\mathrm{Na}, \mathrm{K}$ phosphate $50 \mathrm{mM} \mathrm{pH} \mathrm{8.0,} \mathrm{DTT} 1 \mathrm{mM})$ and supplemented with $20 \mathrm{mM} \mathrm{L-}$ or D-amino acids) was derivatized with L-FDAA (1-fluoro-2-4dinitrophenyl-5-L-alanine amide, Marfey's reagent, Thermo Scientific). First, an equal volume of $\mathrm{NaHCO}_{3} 0.5 \mathrm{M}$ was added to the racemization reaction; then, $6 \mu \mathrm{l}$ of this reaction were reacted with L-FDAA ( $10 \mathrm{mg} / \mathrm{mL}$ in acetone) at $80^{\circ} \mathrm{C}$ for $3 \mathrm{~min}$. The reaction was stopped with 2 $\mathrm{N} \mathrm{HCl}$ and the samples were filtered. The products were separated at $2 \mathrm{~mL} / \mathrm{min}$ with a linear gradient of triethylamine phosphate/acetonitrile in HPLC for 45 min with an Aeris peptide column $(250 \times 4.6 \mathrm{~mm} ; 3.6 \mu \mathrm{m}$ particle size) (Phenomenex, USA) and detected at $340 \mathrm{~nm}$. For the co-incubation with alanine or methionine, the flow was $1 \mathrm{~mL} / \mathrm{min}$ and the gradient length was set to 105 min. Background noise was removed from the L,D-His racemization reactions graphics.

Activity assay followed by TLC

39 Cellular fractions of $S$. aureus WT and cntL mutant strains were supplemented with EDTA and DTT (1 mM final) prior to the TLC assay. $\left[{ }^{14} \mathrm{C}\right]$-SAM (BioTrend, Köln, Germany) was added to the cell fraction(s) at a final concentration of $20 \mu \mathrm{M}$, together with $1 \mathrm{mM}$ pyruvate and $100 \mu \mathrm{M}$ 
purified proteins consisted in incubating the enzymes, either separately or by co-incubation, at a final concentration of $3 \mu \mathrm{M}$ and using $\left[{ }^{14} \mathrm{C}\right]-\mathrm{SAM}(20 \mu \mathrm{M}), \mathrm{NAD}(\mathrm{P}) \mathrm{H}(30 \mu \mathrm{M})$, L- or D-histidine $(10 \mu \mathrm{M})$ and $\alpha$-keto acid (pyruvate or $\alpha$-keto glutarate; $1 \mathrm{mM}$ ). The total volume was $100 \mu \mathrm{L}$ in buffer D (HEPES $50 \mathrm{mM}$, DTT $1 \mathrm{mM}$, EDTA $1 \mathrm{mM}$, pH 9.0). The mixture was incubated for 30 $\min$ at $28{ }^{\circ} \mathrm{C}$. The reactions were stopped by adding ethanol to a final concentration of $50 \%(\mathrm{v} / \mathrm{v})$ and the products were then separated by TLC. An aliquot of $10 \mu \mathrm{L}$ of the reaction mixtures were spotted on HPTLC (High Performance TLC) Silica Gel 60 Glass Plates (Merck KGaA), and the plates were developed with a phenol: $n$-butanol:formate:water $(12: 3: 2: 3 \mathrm{vol} / \mathrm{vol})$ solvent system. These separation parameters were used in the initial biochemical characterization of plant nicotianamine synthase (48). HPTLC plates were dried and exposed to a $\left[{ }^{14} \mathrm{C}\right]$-sensitive imaging plate for 2-3 days. Imaging plates were then scanned on a Typhoon FLA 7000 phosphorimager (GE Healthcare).

Construction of promoter-reporter gene fusion and luciferase assays.

15 The reporter gene luxAB from Vibrio ficheri cloned into shuttle vector pCN58 was used for gene expression analysis. The cntK upstream region (569bp) was cloned using oligonucleotides indicated in Table S1, as previously described for the cntA promoter (10). To follow the cnt promoter-luciferase reporter activity, CDM and LB media were inoculated with an overnight culture to obtain an optical density $\left(\mathrm{OD}_{600}\right)$ of 0.1 or 0.05 , respectively. Culture aliquots were removed, monitored for growth $\left(\mathrm{OD}_{600}\right)$, and submitted to luminescence assays. Measurements of luminescence were performed immediately after mixing $450 \mu \mathrm{l}$ of cell suspension with $50 \mu 1$ of $0.1 \%$ (vol/vol in $40 \%$ ethanol) nonanal (Sigma, St Louis, MO). Luciferase activity, expressed in relative luminescence units (RLUs), was measured using a Biolumat LB9500T Luminometer (Berthold, Bad Wildbad, Germany), based on $10 \mathrm{~s}$ measurements in the integrate data mode. Specific luciferase activities were calculated by dividing the RLUs by absorbance (RLU/OD 600 ). 27 The promoterless pCN58 plasmid was used as control in reporter gene assays. 
1 Supplementary Figures and Table
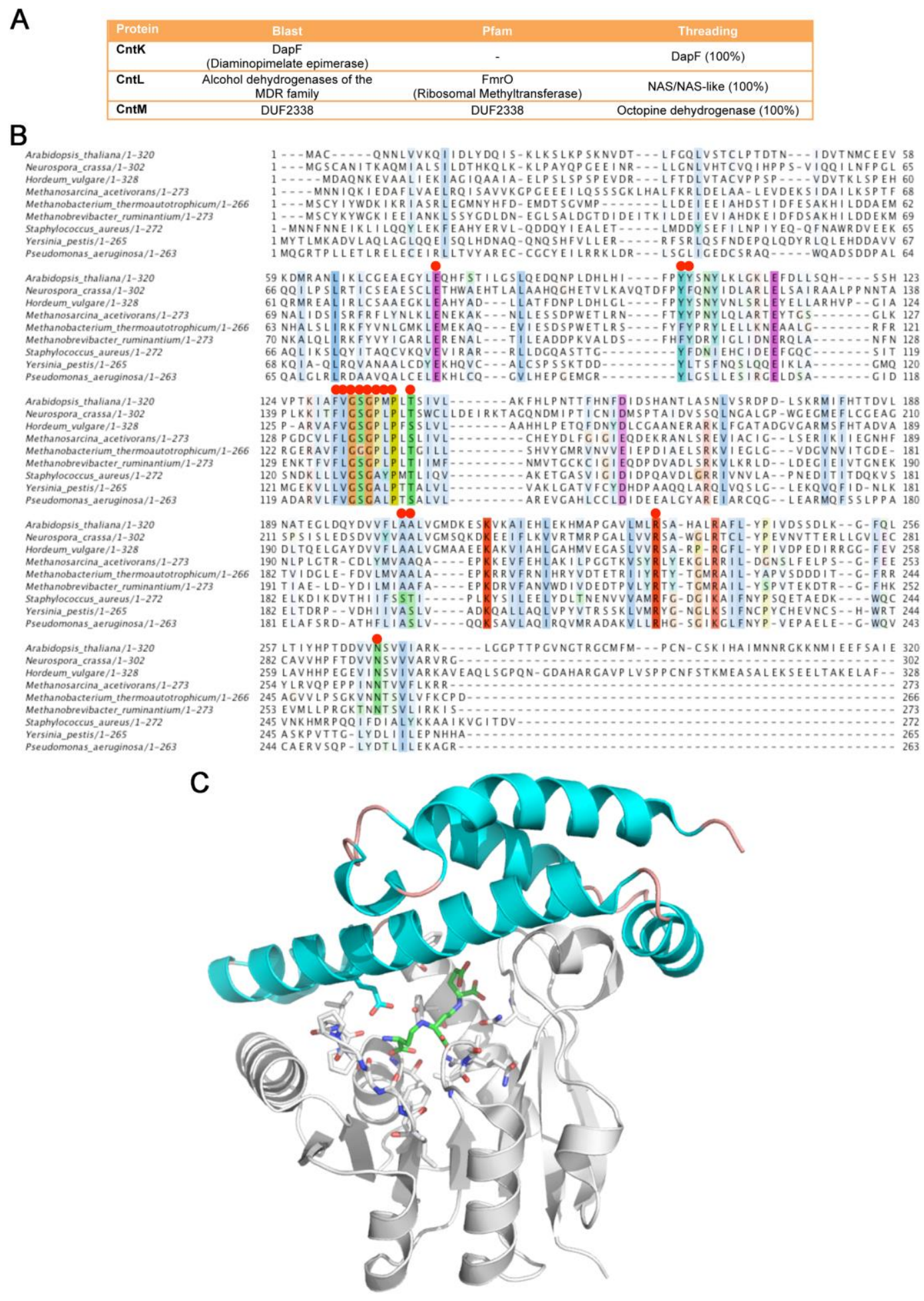

Figure S1: Sequence analysis of the three putative enzymes located in the cnt operon. (A) 4 Summary of sequence analysis of $\mathrm{CntK}$, CntL and CntM protein sequences through PSI-Blast, 5 Pfam and Phyre2. (B) Sequence alignment of three eukaryotic NAS, three archaeal NAS-Like 
1 and three bacterial NAS-Like enzymes, including CntL from S. aureus. Threshold for the 2 ClustalX coloring scheme correspond to $30 \%$ sequence conservation as defined in Jalview. 3 Residues marked by a red dot are conserved residues that are located near the 4 thermonicotianamine (tNA) product in the structure of an archaeal NAS-Like enzyme shown in 5 C. (C) Structure of an archaeal NAS-Like enzyme (pdb code 3FPE) in complex with its product 6 tNA (represented in green stick). The N-Terminal domain is colored in blue (helices) and salmon 7 (loops), and the C-Terminal domain in light gray. Side chains of conserved residues in the 8 sequence alignment (labeled with a red dot in B) are represented in stick. Most of the conserved 9 residues are located closed to the tNA product, therefore clearly indicating that bacterial NAS10 like enzymes are related to the NAS family. 
Staphylococcus aureus Mu50

Pseudomonas aeruginosa PAOI

Yersinia pestis KIM

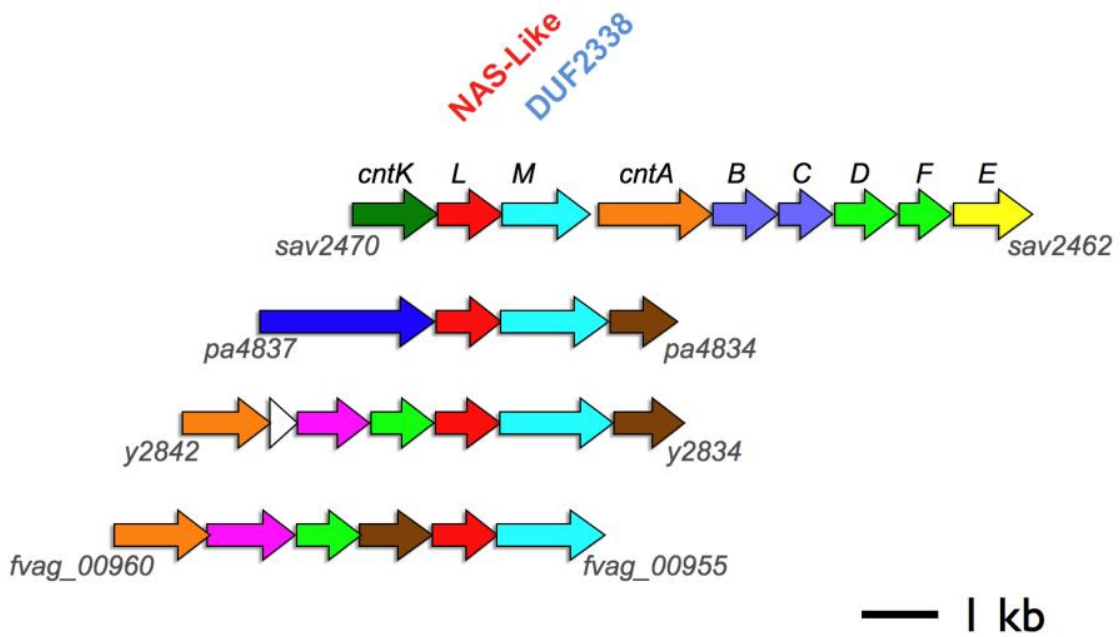

Fusobacterium varium ATCC27725$$
\text { fvag_00960 fvag_00955 }
$$



EamA

\section{Solute Binding Protein}

ATPase

Permease: BPD_transp / FecCD

Epimerase

Figure S2: Synteny of the NAS-like encoding gene in selected bacteria. Genes are colorcoded as a function of the Pfam family to which the product belongs, as indicated in the grey inset. $Y$. pestis, $P$. aeruginosa and $F$. varium does not possess a close CntK homologue but a distantly related protein that is annotated as diaminopymelate epimerase (DapF). 



2 Figure S3: Metabolomic study of $E$. coli strains expressing the cnt operon or the empty 3 vector. (A) HILIC/ICP-MS chromatograms of intracellular fractions of $E$. coli strain 4 (supplement with $0.1 \mu \mathrm{M}$ nickel to ensure a nickel complexation of the metallophore) and 5 expressing the cht operon from $S$. aureus (blue line) and comparison with the strain expressing 6 the empty vector (red line). (B) Mass spectra of a chelating compound present in its complexed 7 forms with nickel. Molecular formula was deduced from the exact mass of the ${ }^{58} \mathrm{Ni}$ complex. (C) 8 HILIC/ESI-MS extracted ion chromatograms of $\mathrm{m} / \mathrm{z}$ 385.06. 
A

Cloning, expression \& purification of the SBP (CntA) from E. coli

Incubation in [(supernatant of $S$. aureus cntA

Re-Purification of CntA

Buffer exchange

Mass spectrometry purification. mutant grown in $\mathrm{CDM}$ ) $+\mathrm{Ni}]$



Figure S4: Fishing of the metallophore through a knowledge based approach. (A) Scheme of the "knowledge based approach". (B) Final mass spectra of CntA after incubation and re- 
A

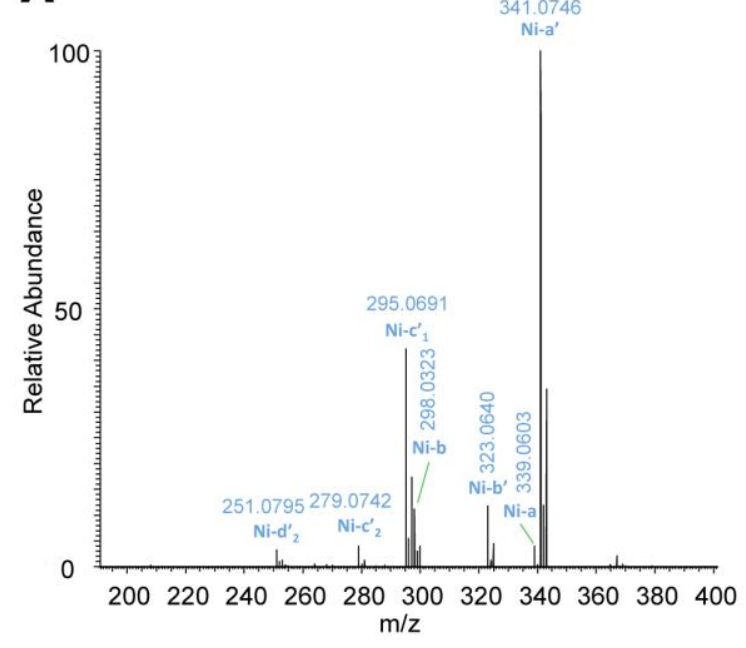

B

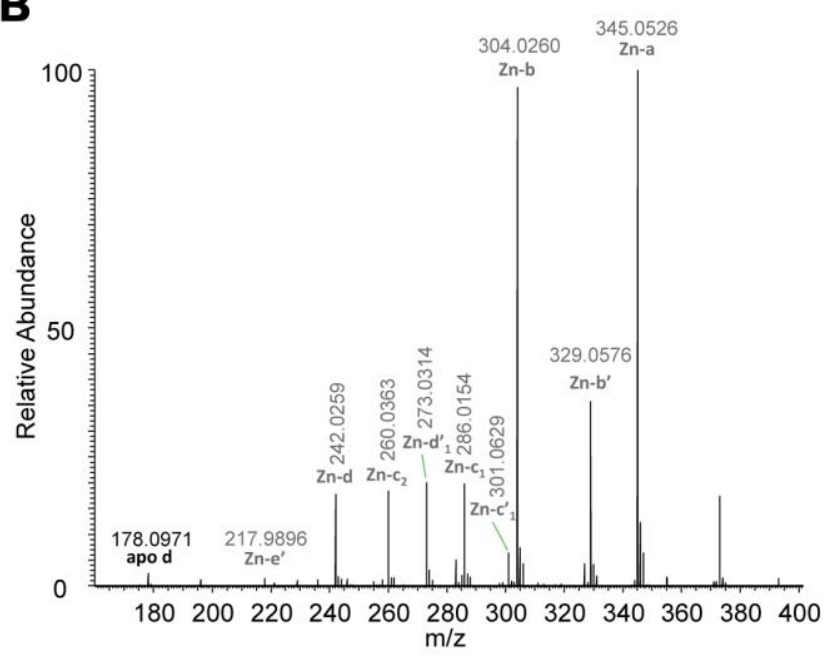

C

Theoretical Mw of the $\mathrm{Zn}$ complex Theoretical Mw of the Ni complex Theoretcal Mw of apo form C

a

<smiles>CCC(C)CN</smiles>

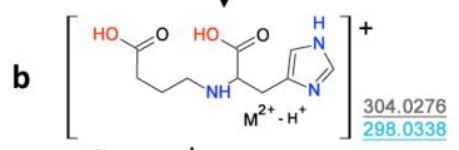
$-\mathrm{H}_{2} \mathrm{O} \downarrow \downarrow-\mathrm{CO}_{2}$

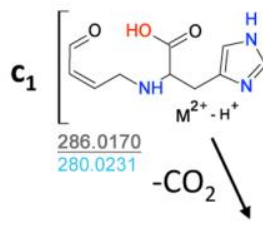
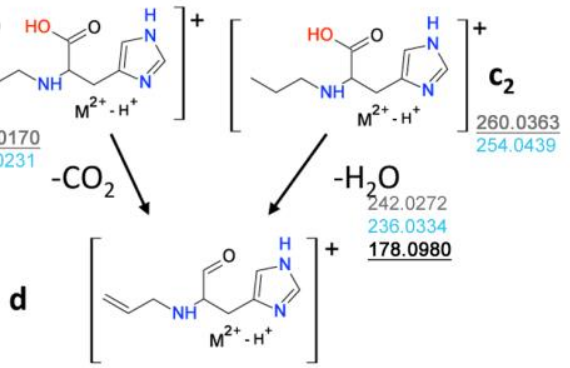

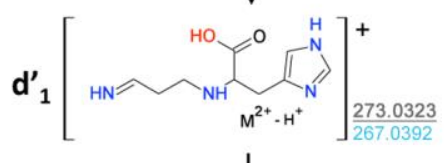
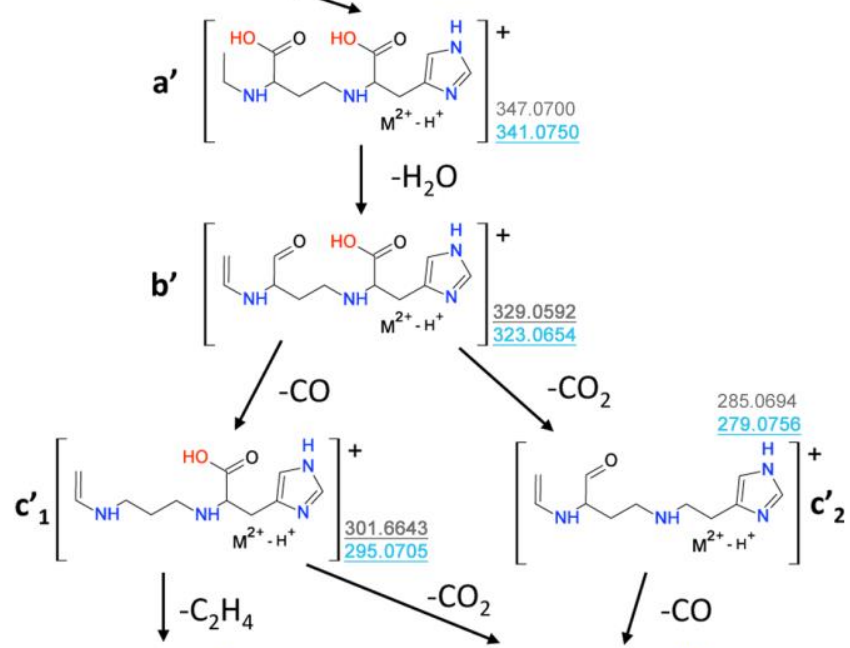

$\downarrow-\mathrm{H}_{2} \mathrm{O}$

1.0750
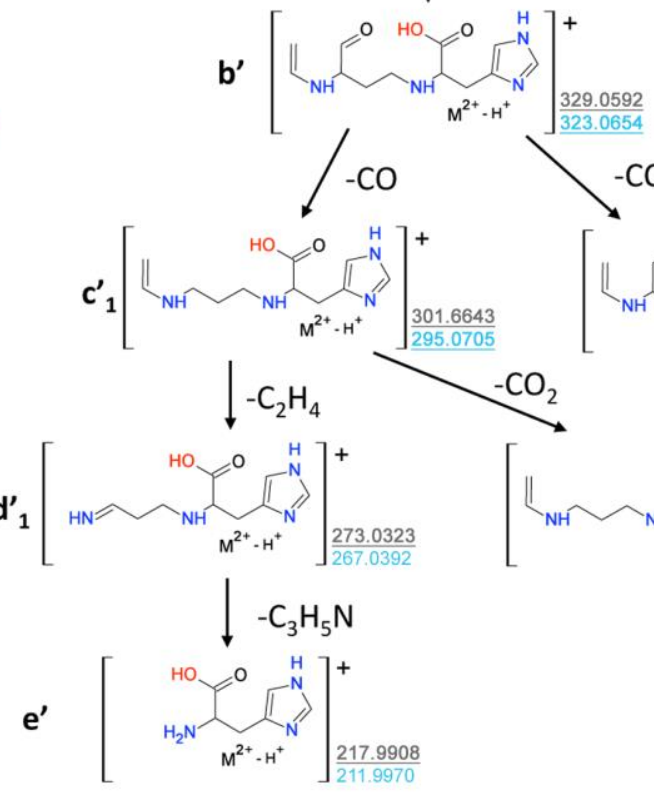

Figure S5: MS/MS fragmentation of Staphylopine-Metal complexes. MS/MS fragmentation of Staphylopine-Zn (A) and Staphylopine-Ni complexes (B). The mass of the ions are indicated as well as their interpretation with the fragmentation scheme shown in (C).

(C) Proposed 
1 fragmentation scheme of Staphylopine-Zn and Staphylopine-Ni complexes. The theoretical 2 masses of the ions are indicated on the right of the proposed ion. The underlined masses were 3 detected in the observed fragmentation pattern shown in (A) and (B). 


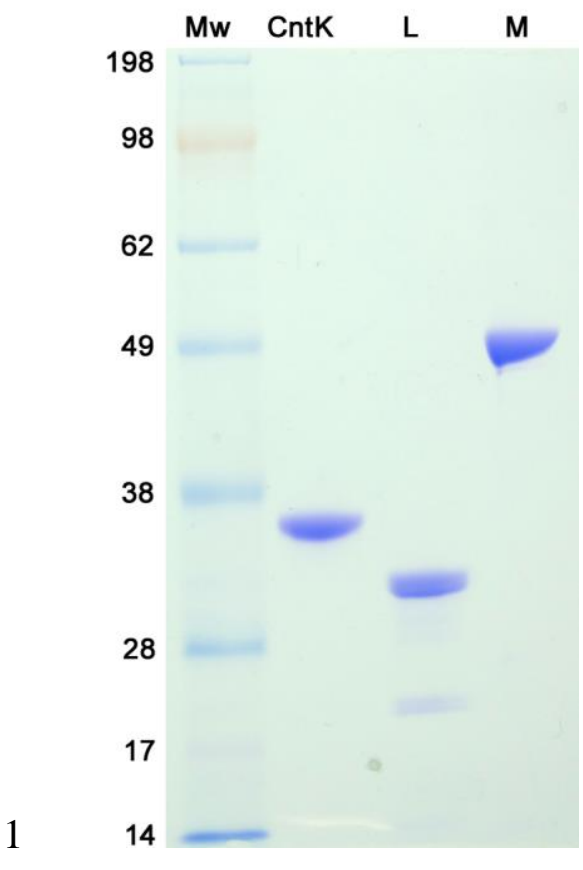

2 Figure S6: SDS-PAGE gel of purified CntK, CntL and CntM. The expected molecular 3 weights are $35 \mathrm{kDa}, 31 \mathrm{kDa}$ and $51 \mathrm{kDa}$ for $\mathrm{CntK}$, CntL and CntM respectively. The final 4 purification yields are 2, 5 and $20 \mathrm{mg}$ of pure protein per liter of culture. 

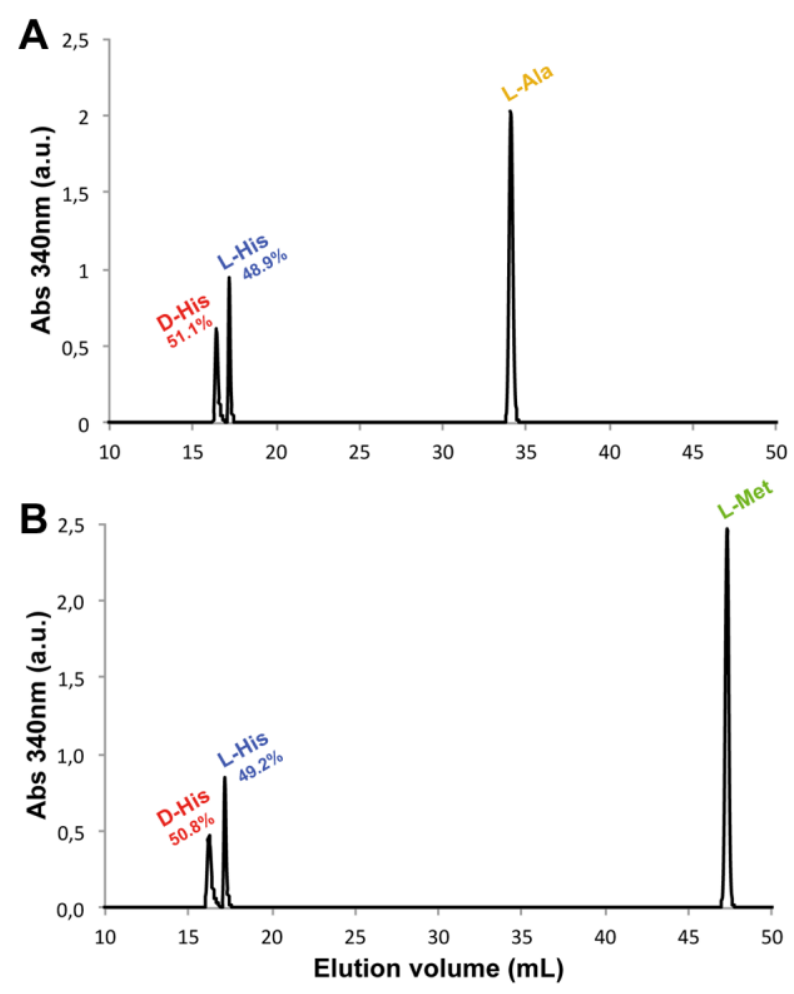

Figure S7: Activity of CntK in the presence of competing amino acids. (A) CntK was incubated with $20 \mathrm{mM}$ of L-histidine and $40 \mathrm{mM}$ of L-alanine. (B) CntK was incubated with 20 $\mathrm{mM}$ of L-histidine and $40 \mathrm{mM}$ of L-methionine. 
A

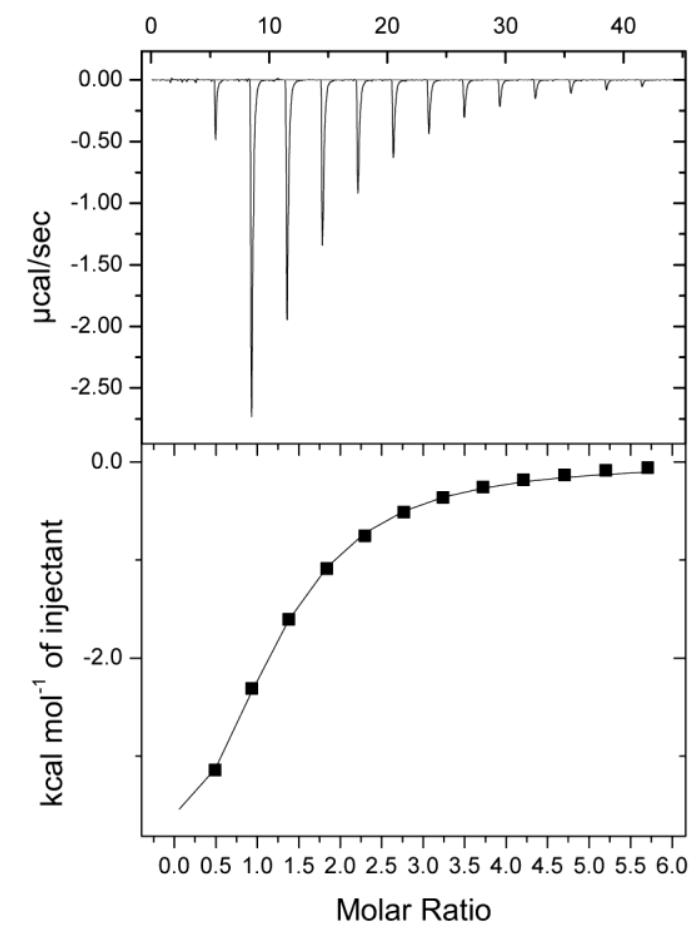

B

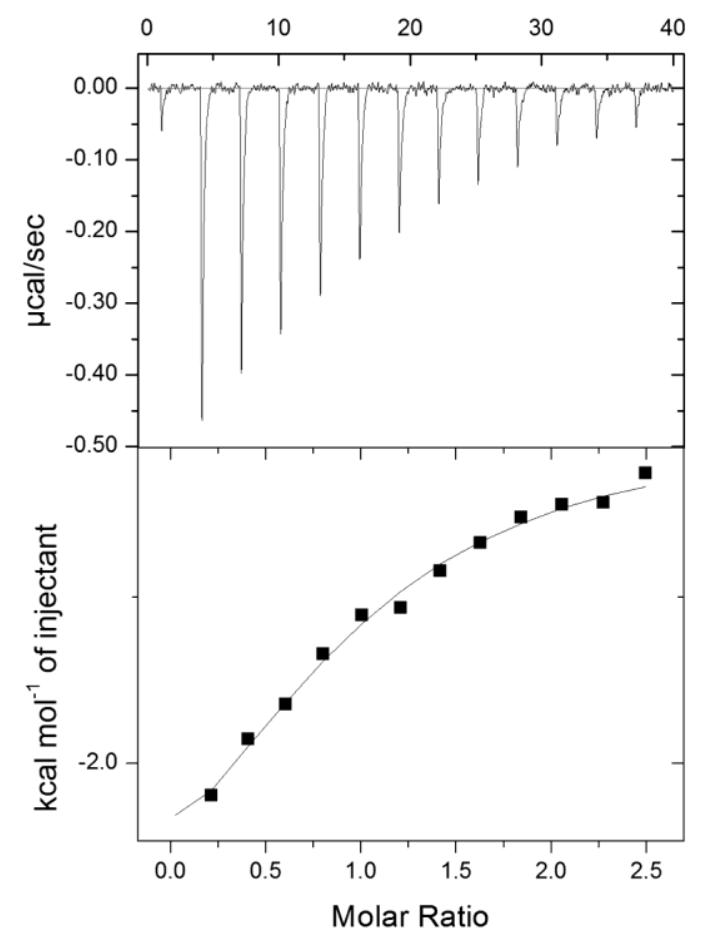

Figure S8: Microcalorimetric titration of CntL with SAM and CntM with NADPH. (A) Representative data (raw data on top and curve fit below) from an ITC experiment where SAM was titrated into the reaction cell containing CntL. The first addition (not used in the fit) was half the volume of the other additions. Thermodynamic values obtained from the curve fit are: $\Delta S=$ $1.29 \mathrm{cal} / \mathrm{mol} / \mathrm{K}, \Delta H=-5212 \pm 211.5 \mathrm{cal} / \mathrm{mol}, K_{\mathrm{d}}=68 \pm 5 \mu \mathrm{M}, n=1.07 \pm 0.03$, where $n$ is the stoichiometry of the binding. (B) Representative data (raw data on top and curve fit below) from an ITC experiment where NADPH was titrated into the reaction cell containing CntM. The first addition (not used in the fit) was half the volume of the other additions. Thermodynamic values obtained from the curve fit are: $\Delta S=6.91 \mathrm{cal} / \mathrm{mol} / \mathrm{K}, \Delta H=-3831 \pm 530.1 \mathrm{cal} / \mathrm{mol}, K_{\mathrm{d}}=48 \pm 4$

$11 \mu \mathrm{M}, n=0.929 \pm 0.09$, where $n$ is the stoichiometry of the binding. 

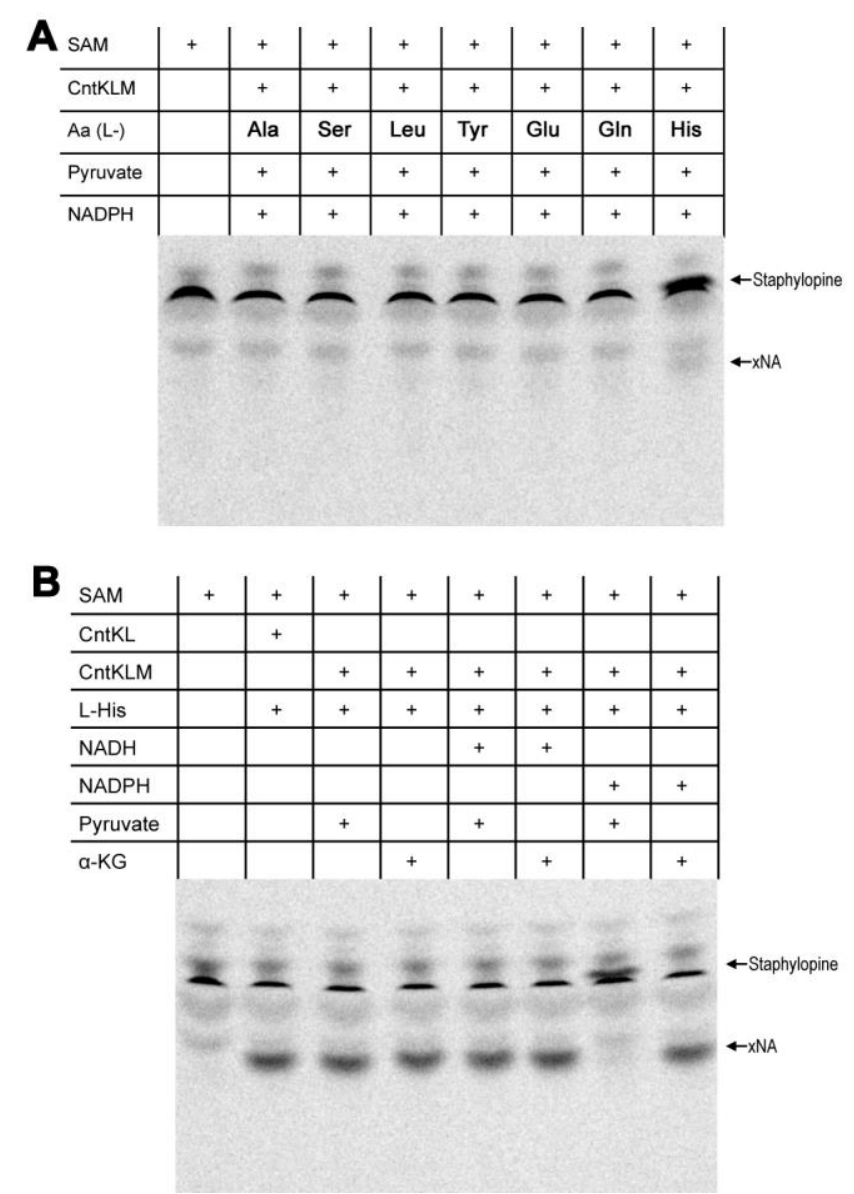

2 Figure S9: Substrate specificity of CntK, CntL and CntM. (A) Test of seven different L3 aminoacids with divers properties on the activity of CntKLM using NADPH and pyruvate as 4 substrates of CntM. (B) Test of substrate specificity of CntM using all three biosynthetic 5 enzymes and different CntM substrates (NADPH $v s$ NADH and pyruvate $v s$-keto glutarate; $\alpha$ 6 KG). 

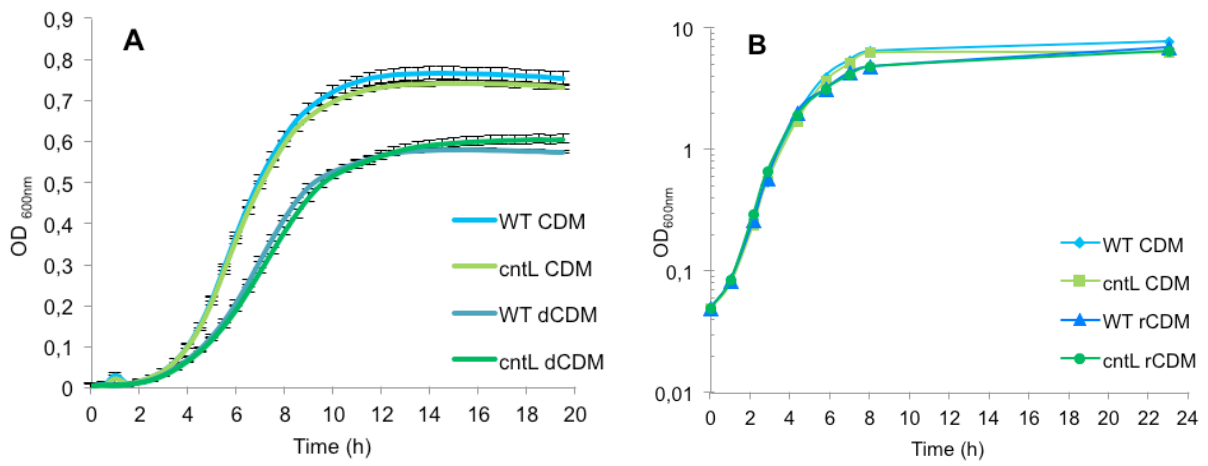

2 Figure S10: Growth curves of the WT and cntL strains of $\boldsymbol{S}$. aureus. (A) in CDM and 3 demetalated CDM (dCDM) in microplates, or (B) in CDM and demetalated-NTA CDM with 4 metalated NTA resin $(\mathrm{rCDM})$ in $50 \mathrm{ml}$ tubes at $37^{\circ} \mathrm{C}$ with aeration. 

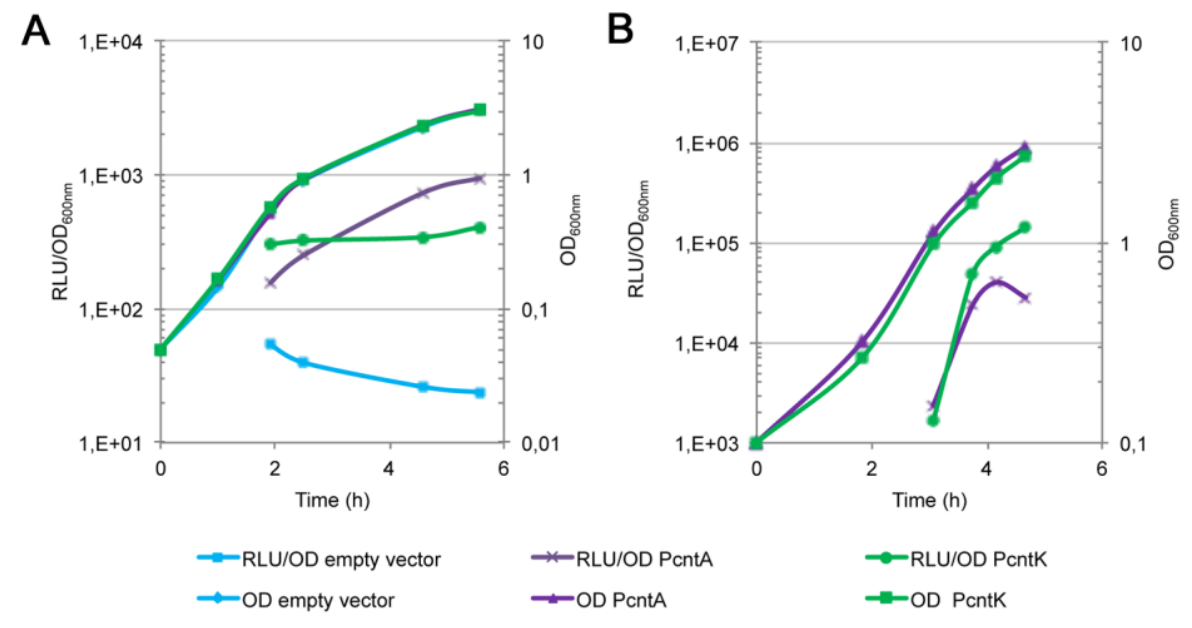

C
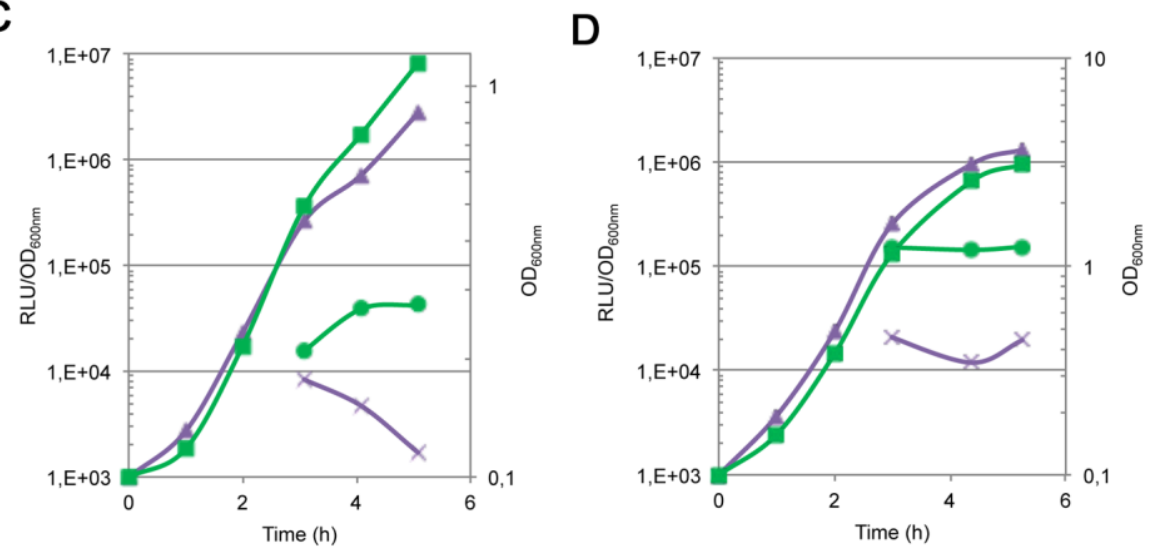

Figure S11: Luciferase activities of WT strain harbouring fusion of PcntA-luxAB (PcntA) and PcntK-luxAB (PcntK) in various media. (A) LB medium. (B) CDM. (C) dCDM. (D) 4 rCDM. 

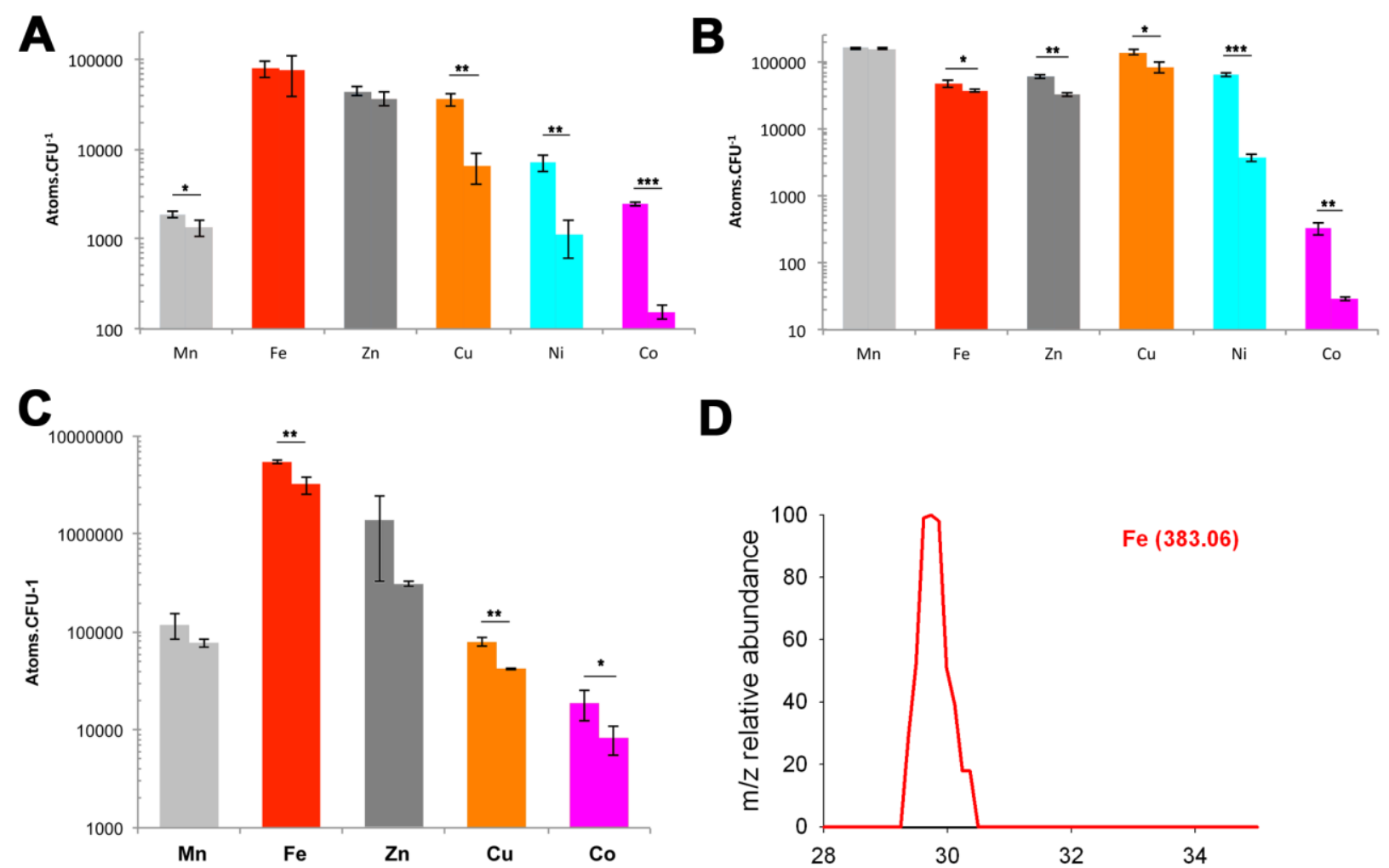

D

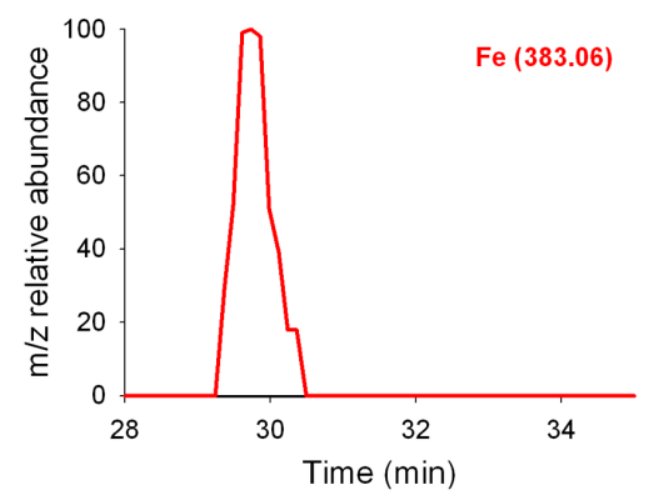

Figure S12: Import of divalent metals in various culture conditions. (A) Intracellular metal content determined by ICP-MS of $S$. aureus WT (left bars) and cntL (right bars) mutant strains grown in dCDM. Error bars, mean \pm s.d. $* P<0.05$ and $* * P<0.01$. (B) Intracellular metal content determined by ICP-MS of $S$. aureus WT (left bars) and cntL (right bars) mutant strains grown in rCDM. Error bars, mean \pm s.d. $* P<0.05, * * P<0.01$ and $* * * P<0.001$. (C) Intracellular metal content determined by ICP-AES of $S$. aureus WT (left bars) and $c n t L$ (right bars) mutant strains grown in LB medium without further metal supplementation. Error bars, mean \pm s.d. $* P<0.05$ and $* * P<0.01$. (D) HILIC/ESI-MS extracted ion chromatograms (XIC) of the Staphylopine-Fe complex detected in the extracellular fraction of $S$. aureus grown in CDM. Chromatogram was traced using the exact mass of the complex ( $\pm 3 \mathrm{ppm}$ ). This mass was absent in the extracellular 12 fraction of the $c n t L$ mutant. 


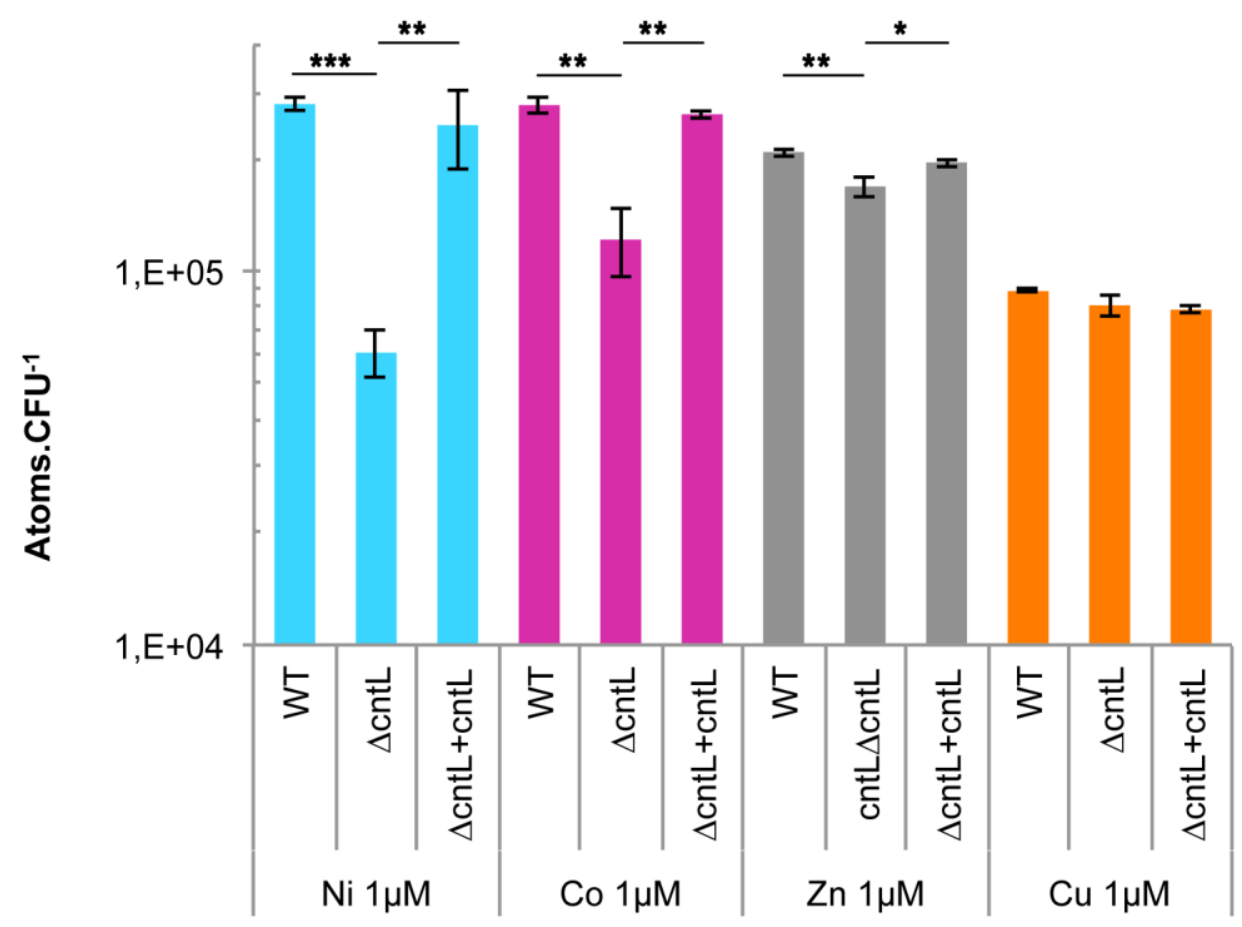

Figure S13: Metal content of WT S. aureus, cntL mutant and complemented strains. Cells were grown in CDM supplemented with the indicated metal $(1 \mu \mathrm{M})$, and subsequently grown overnight, prior to determination of their intracellular metal concentration by ICP-AES. Error bars, mean \pm s.d. $* * P<0.01$ and $* * * P<0.001$. 

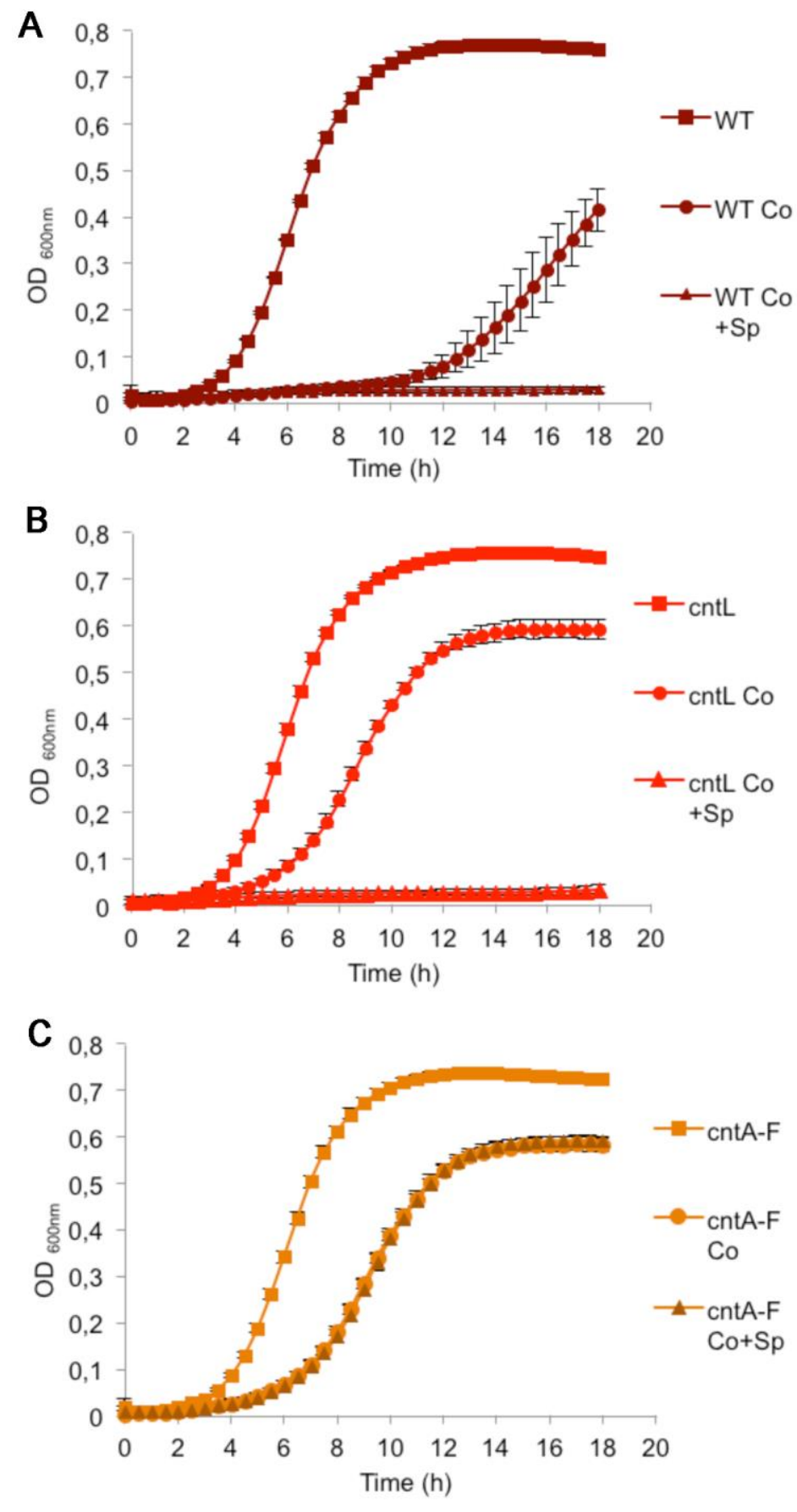

2 Figure S14: Toxicity of cobalt and CntA-F / staphylopine-dependent transport of cobalt.

3 (A) WT, (B) $c n t L$ and (C) $c n t A-F$ strains were grown at $37^{\circ} \mathrm{C}$ in microplates in $\mathrm{CDM}$ 4 supplemented or not with $2 \mathrm{mM}$ cobalt $(\mathrm{Co}), 10 \mu \mathrm{M}$ of staphylopine (Sp), or both $(\mathrm{Co}+\mathrm{Sp})$. 5 Growth in CDM +/- Sp alone was similar for all strains, indicating that staphylopine alone is not 6 toxic or beneficial in these conditions. 
A

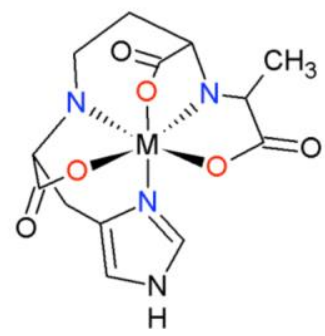

B

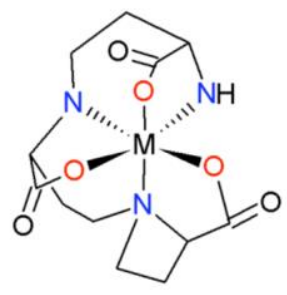

C

\begin{tabular}{lcc} 
& \multicolumn{2}{c}{ pKd } \\
\cline { 2 - 3 } & Nicotianamine (49) & Staphylopine (this study) \\
\hline $\mathrm{Cu}^{2+}$ & 18.6 & 19.0 \\
$\mathrm{Ni}^{2+}$ & 16.1 & 16.4 \\
$\mathrm{Co}^{2+}$ & 14.8 & 15.1 \\
$\mathrm{Zn}^{2+}$ & 14.7 & 15.0 \\
$\mathrm{Fe}^{2+}$ & 12.1 & 12.3 \\
$\mathrm{Mn}^{2+}$ & 8.8 & 9.1
\end{tabular}

2 Figure S15: Putative structure and estimated pKd for staphylopine by comparison with 3 nicotianamine. Putative chemical structure of staphylopine-metal complex (A) and 4 nicotianamine-metal complex (B). (C) pKd values for staphylopine complexes were estimated by 5 metal competition experiments with nicotianamine by ESI MS. 


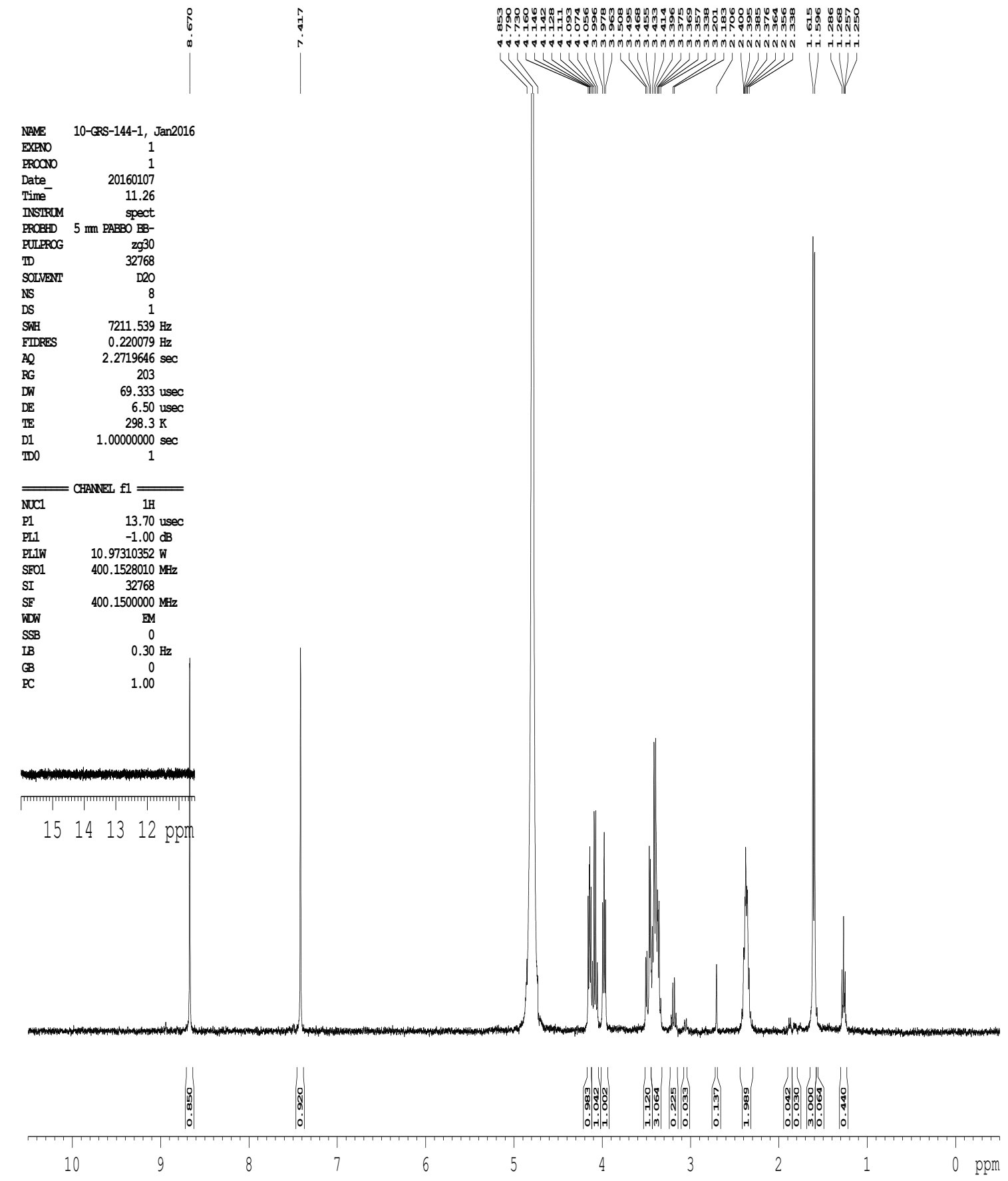

2 Figure S16: NMR spectrum of synthetic staphylopine. 


\begin{tabular}{|c|c|c|}
\hline Name & Sequence (5'-3') & $\begin{array}{l}\text { Targeted } \\
\text { plasmid }\end{array}$ \\
\hline CntA-Fw & CACCATGAGAAAACTAACTAAAATG & \multirow[t]{2}{*}{ pET100 } \\
\hline CntA-Rv & TTATTTATACTGCATTTCATTGAA & \\
\hline CntK-Fw & CACCATGAATCGGCAGGTTATAGA & \multirow[t]{2}{*}{ pET100 } \\
\hline CntK-Rv & TTATTCTATATATGCTTTTCCTGT & \\
\hline CntL-Fw & AATAACTTTAATAATGAAATCAAA & \multirow{2}{*}{$\begin{array}{l}\text { pET } \\
\text { SUMO }\end{array}$} \\
\hline CntL-Rv & TTAGACATCCGTAATACCTACCTTTATA & \\
\hline CntM-Fw & CACCATGTCTAAATTATTAATGATAGGCAC & \multirow[t]{2}{*}{ pET101 } \\
\hline CntM-Rv & TGAAAGCGTTCTATTGATTTCCA & \\
\hline Cntop-Fw & $\begin{array}{l}\text { AGAAGGAGATATACATATGAATCGGCAGGTTATAG } \\
\text { AATTTTCT }\end{array}$ & \multirow[t]{2}{*}{ pET22b+ } \\
\hline Cnt & $\begin{array}{l}\text { GGTGGTGGTGCTCGAGAAGACTACTCGCTGGACGT } \\
\text { GGTGTT }\end{array}$ & \\
\hline Mut-cntL5'A & CGGGATCCTGGCCGCAACACATGTATGCTGTG & pMAD \\
\hline Mut-cntL3'A & $\begin{array}{l}\text { CATCCGTAATACCTACAAAGTTATTCATTGTAGGCT } \\
\text { CCTTATTC }\end{array}$ & pMAD \\
\hline Mut-cntL5'B & $\begin{array}{l}\text { TACAATGAATAACTTTGTAGGTATTACGGATGTCTA } \\
\text { AAT }\end{array}$ & pMAD \\
\hline Mut-cntL3'B & CGGGATCCGCTAAAGCAGAGATTCGTTGACAC & pMAD \\
\hline Mut-cntE-5'A & CGCGGATCCTGTTGGAAGAAGTCGGTCTATC & pMAD \\
\hline Mut-cntE-3'A & ACTACTCGCTCAAGCCATTGCACCTTTCAT & pMAD \\
\hline Mut-cntE-5'B & CGAGTAGTCTTTAATGAA & pMAD \\
\hline Mut-cntE-3'B & TCCCCCCGGGTAATACATGGTGGGAATTCATTCC & pMAD \\
\hline pOS-cntL5 & CGCGGATCCGCCTACAATGAATAACTTTAATAATG & pOS1-Plgt \\
\hline pOS & CGCGGATCCGGATTGCGACCGGACCAGTGC & pOS1-Plgt \\
\hline pLUX-cntK5 & CGCGGATCCGAAGTTTATGGAAGGATTATC & pCN58 \\
\hline pLUX-cntK3 & CCGGAATTCCGACACTCCTTTAGATGTA & pCN58 \\
\hline
\end{tabular}

Table S1: Oligonucleotides used in this study 


\begin{tabular}{|c|c|}
\hline Ingredient & Final medium concentration $(\mathrm{g} / \mathrm{l})$ \\
\hline D-glucose & 4 \\
\hline \multicolumn{2}{|l|}{ Amino acids } \\
\hline L-cysteine & 0,48 \\
\hline L-aspartic acid & 2,4 \\
\hline L-glutamic acid & 2,4 \\
\hline L-proline & 2,4 \\
\hline L-arginine & 0,36 \\
\hline L-glycine & 2,4 \\
\hline L-histidine & 0,48 \\
\hline L-lysine HCL & 0,6 \\
\hline L-serine & 2,4 \\
\hline L-valine & 0,48 \\
\hline L-threonine & 2,4 \\
\hline L-alanine & 2,4 \\
\hline L-isoleucine & 0,6 \\
\hline L-leucine & 0,6 \\
\hline L-tryptophan & 0,06 \\
\hline L-methionine & 0,18 \\
\hline L-tyrosine & 0,18 \\
\hline L-phenylalanine & 0,2 \\
\hline \multicolumn{2}{|l|}{ Salts } \\
\hline $\mathrm{Na}_{2} \mathrm{HPO}_{4}$ & 5,7 \\
\hline$(\mathrm{NH} 4)_{2} \mathrm{SO}_{4}$ & 6,84 \\
\hline $\mathrm{KH}_{2} \mathrm{PO}_{4}$ & 1,34 \\
\hline $\mathrm{MgSO}_{4} 7 \mathrm{H}_{2} \mathrm{O}$ & 0,247 \\
\hline \multicolumn{2}{|l|}{ Vitamins } \\
\hline Nicotinic acid & 0,01194 \\
\hline Thiamine HCL & 0,0006 \\
\hline Ca panthothenate & 0,002 \\
\hline Biotine & 0,0001 \\
\hline
\end{tabular}

1 Table S2: Composition of chemically defined medium. The $\mathrm{pH}$ is adjusted to 7 with $\mathrm{KOH}$ and 2 the volume to $1 \mathrm{~L}$ with milliQ water. The medium is then sterilized by filtration through a $3 \quad 0,22 \mu \mathrm{m}$ filter. 


\begin{tabular}{ccccccc} 
& \multicolumn{7}{c}{ Culture media concentration, nM } \\
& $\mathrm{Mn}$ & $\mathrm{Fe}$ & $\mathrm{Zn}$ & $\mathrm{Cu}$ & $\mathrm{Ni}$ & $\mathrm{Co}$ \\
\hline $\mathbf{L B}$ & $116 \pm 9$ & $4450 \pm 356$ & $5320 \pm 426$ & $206 \pm 16$ & $25 \pm 2$ & $134 \pm 11$ \\
$\mathbf{C D M}$ & $17 \pm 3$ & $171 \pm 14$ & $105 \pm 8$ & $7 \pm 2$ & $12 \pm 2$ & $<5$ \\
dCDM & $<5$ & $20 \pm 3$ & $22 \pm 3$ & $<5$ & $<5$ & $<5$ \\
\hline
\end{tabular}

1

Table S3: Metal concentrations in culture media and in the intracellular fraction of WT 3 cells in different culture conditions. 


\section{Supplementary references}

2 34. S. F. Altschul et al., Nucleic Acids Res 25, 3389 (1997).

3 35. R. D. Finn et al., Nucleic Acids Res 42, D222 (2014).

4 36. R. C. Edgar, Nucleic Acids Res 32, 1792 (2004).

5 37. M. Clamp, J. Cuff, S. M. Searle, G. J. Barton, Bioinformatics 20, 426 (2004).

638 L. A. Kelley, S. Mezulis, C. M. Yates, M. N. Wass, M. J. Sternberg, Nat Protoc 10, 845 7 (2009).

8 39. D. Vallenet et al., Nucleic Acids Res 41, D636 (2013).

9 40. A. Hiron, E. Borezee-Durant, J. C. Piard, V. Juillard, J Bacteriol 189, 5119 (2007).

10 41. M. Arnaud, A. Chastanet, M. Debarbouille, Appl Environ Microbiol 70, 6887 (2004).

11 42. C. Rigoulay et al., Infect Immun 73, 563 (2005).

12 43. J. Bubeck Wardenburg, W. A. Williams, D. Missiakas, Proc Natl Acad Sci U S A 103, $1313831(2006)$.

14 44. D. Taylor, K. T. Holland, J Appl Bacteriol 66, 319 (1989).

15 45. L. Grillet et al., J Biol Chem 289, 2515 (2014).

16 46. L. Ouerdane, S. Mari, P. Czernic, M. Lebrun, R. Lobinski, Journal of Analytical Atomic

17 Spectrometry 21, 676 (2006).

18 47. N. von Wiren et al., Plant Physiol 119, 1107 (1999).

19 48. K. Higuchi, K. Kanazawa, N. K. Nishizawa, S. Mori, Plant and Soil 178, 171 (1996).

20 49. I. Benes, S. K., H. Ripperger, A. Kircheiss, Experientia 39, 261 (1983). 